\title{
Spacetime from unentanglement
}

\author{
Yasunori Nomura, ${ }^{1,2,3}$ Pratik Rath, ${ }^{1,2}$ and Nico Salzetta ${ }^{1,2}$ \\ ${ }^{1}$ Berkeley Center for Theoretical Physics, Department of Physics, University of California, \\ Berkeley, California 94720, USA \\ ${ }^{2}$ Theoretical Physics Group, Lawrence Berkeley National Laboratory, California 94720, USA \\ ${ }^{3}$ Kavli Institute for the Physics and Mathematics of the Universe (WPI), \\ University of Tokyo, Kashiwa, Chiba 277-8583, Japan
}

(Received 9 February 2018; published 16 May 2018)

\begin{abstract}
The past decade has seen a tremendous effort toward unraveling the relationship between entanglement and emergent spacetime. These investigations have revealed that entanglement between holographic degrees of freedom is crucial for the existence of bulk spacetime. We examine this connection from the other end of the entanglement spectrum and clarify the assertion that maximally entangled states have no reconstructable spacetime. To do so, we first define the conditions for bulk reconstructability. Under these terms, we scrutinize two cases of maximally entangled holographic states. One is the familiar example of AdS black holes; these are dual to thermal states of the boundary conformal field theory. Sending the temperature to the cutoff scale makes the state maximally entangled and the respective black hole consumes the spacetime. We then examine the de Sitter limit of Friedmann-Robertson-Walker (FRW) spacetimes. This limit is maximally entangled if one formulates the boundary theory on the holographic screen. Paralleling the anti-de Sitter (AdS) black hole, we find the resulting reconstructable region of spacetime vanishes. Motivated by these results, we prove a theorem showing that maximally entangled states have no reconstructable spacetime. Evidently, the emergence of spacetime is endemic to intermediate entanglement. By studying the manner in which intermediate entanglement is achieved, we uncover important properties about the boundary theory of FRW spacetimes. With this clarified understanding, our final discussion elucidates the natural way in which holographic Hilbert spaces may house states dual to different geometries. This paper provides a coherent picture clarifying the link between spacetime and entanglement and develops many promising avenues of further work.
\end{abstract}

DOI: 10.1103/PhysRevD.97.106010

\section{INTRODUCTION}

It is believed that dynamical spacetime described by general relativity is an emergent phenomenon in the fundamental theory of quantum gravity. Despite this pervasive idea, the materialization of spacetime itself is not fully understood. Holography posits that a fundamental description of quantum gravity resides in a nongravitational spacetime whose dimension is less than that of the corresponding bulk spacetime [1-3]. In this paper, we study the emergence of gravitational spacetime in the context of holography, using the renowned anti-de Sitter (AdS)/conformal field theory (CFT) correspondence [4] and a putative holographic theory of Friedmann-RobertsonWalker (FRW) spacetimes [5].

Published by the American Physical Society under the terms of the Creative Commons Attribution 4.0 International license. Further distribution of this work must maintain attribution to the author(s) and the published article's title, journal citation, and DOI. Funded by SCOAP .
In this paper, we expound on the intimate relationship between the emergence of spacetime and the lack of maximal entanglement in the boundary state. Through this, we see that the existence of spacetime is necessarily nongeneric and that nature seizes the opportunity to construct local spacetime when states deviate from maximal entanglement. A reason why this viewpoint is not heavily emphasized (see, however, e.g., Refs. [6,7]) in the standard context of AdS/CFT is that one almost always considers states with energy much lower than the cutoff (often sent to infinity). The restriction to these "low energy" states implicitly narrows our perspective to those automatically having nonmaximal entropy. However, in a holographic theory with a finite cutoff scale (or a fundamentally nonlocal theory), the regime of maximal entropy is much more readily accessible. This happens to be the case in FRW holography, and perhaps holography in general. Through this lens, we analyze the emergence of spacetime both in the familiar setting of Schwarzschild-AdS spacetime with an infrared cutoff and in flat FRW universes. We explicitly see that the directly reconstructable region of spacetime $[6,8]$ emerges 
only as we deviate from maximally entangled states. This implies that a holographic theory of exact de Sitter space cannot be obtained as a natural limit of theories dual to FRW spacetimes by sending the fluid equation of state parameter, $w$, to -1 . In addition to analyzing these two examples, we prove a theorem demonstrating the lack of directly reconstructable spacetime in the case that a boundary state is maximally entangled.

After surveying the relationship between spacetime and (the lack of) entanglement, we then analyze the deviation from maximal entropy itself. The size of the subregions for which deviations occur reveals valuable information about the underlying holographic theory, and observing the corresponding emergence of spacetime in the bulk provides a glimpse into the mechanism by which nature creates bulk local degrees of freedom. In the case of Schwarzschild-AdS spacetime, reconstructable spacetime (the region between the horizon and the cutoff) appears as the temperature in the local boundary theory (the CFT) is lowered, and the resulting entanglement entropy structure (calculated holographically) is consistent with a local theory at high temperature. However, this entanglement structure is not observed in the case of FRW spacetimes as we adjust $w$ away from -1 ; additionally, the reconstructable region grows from the deepest points in the bulk outward. This suggests that the manner in which entanglement is scaffolded is unlike that of AdS/CFT. In fact, this aberrant behavior leads us to believe that the holographic theory dual to FRW spacetimes has nonlocal interactions.

The relationship between spacetime and quantum entanglement between holographic degrees of freedom is no secret [9-15], but what is spacetime? Undoubtedly, entanglement is a necessity for the existence of spacetime. But it is indeed possible to have too much of a good thing. The analysis here exposes the inability to construct spacetime from maximally entangled boundary states. Since typical states in a Hilbert space are maximally entangled [16], this implies that states with bulk dual are not typical. We see that spacetime is an emergent property of nongeneric states in the Hilbert space with both nonvanishing and nonmaximal entanglement for subregions. The existence of entanglement allows for the construction of a code subspace of states [17] in which local, semiclassical bulk degrees of freedom can be encoded redundantly. Simultaneously, the lack of maximal entanglement allows for a code subspace with subsystem recovery-hence partitioning the bulk into a collection of local Hilbert spaces. With this perspective, we see that holographic theories are exceedingly enterprising — once deviating from maximal entanglement, nature immediately seizes the opportunity to construct spacetime. In this sense, spacetime is the byproduct of nature's efficient use of intermediate entanglement to construct codes with subsystem recovery.

For a given spacetime with a holographic boundary, one can calculate the von Neumann entropies for all possible subregions of the boundary via the Hubeny-RangamaniRyu-Takayanagi (HRRT) prescription $[9,10,15]$. The corresponding entanglement structure heavily constrains the possible boundary states, but by no means uniquely specifies it. In fact, given an entanglement structure and a tensor product Hilbert space, one can always find a basis for the Hilbert space in which all basis states have the desired entanglement structure. If one considers each of these basis states to be dual to the spacetime reproducing the entanglement, then by superpositions one could entirely change the entanglement structure, and hence the spacetime. This property naturally raises the question of how the boundary Hilbert space can accommodate states dual to different semiclassical geometries. Fortunately, for generic dynamical systems, the Hilbert space can be binned into energy bands, and canonical typicality provides us with the result that generic states within these bands have the same entanglement structure, regardless of the energy band's size. This allows the holographic Hilbert space to contain states dual to many different spacetimes, each of which can have bulk excitations encoded state independently. Importantly, this is contingent on the result that typical states have no spacetime.

\section{A. Outline}

Section II walks through the statement that maximally entangled (and hence typical) states have no reconstructable spacetime. This is broken down into parts. First, we must define what we mean by reconstructable; this is detailed in Sec. II A, and is very important for understanding the framework of the rest of the paper. We then use this construction in Sec. II B to investigate the reconstructable region of AdS spacetime with a black hole. We see the expected behavior that the reconstructable region of spacetime vanishes as the temperature of the black hole reaches the cutoff scale, making the state typical. In Sec. II C, we show that de Sitter states are maximally entangled by finding their HRRT surfaces. In Sec. II D, we combine numerical results for flat FRW universes and use the additional property that de Sitter's HRRT surfaces lie on a null cone to show that the reconstructable region vanishes in the de Sitter limit of FRW spacetimes. Motivated by these results, in Sec. II E we prove a theorem showing that if a state is maximally entangled, then its HRRT surfaces either wrap the holographic space or live on the null cone. This is then used to present the general argument that maximally entangled states have no spacetime.

Section III compares the emergence of spacetime in the two theories we are considering. Sections III A and III B present results comparing the entanglement structure of AdS black holes and FRW spacetimes, respectively. Section III C interprets these results and argues that the appropriate holographic dual of FRW spacetimes is most likely nonlocal. 
In Sec. IV, we put together all of the previous results and explain how one Hilbert space can contain states dual to many different semiclassical spacetimes. Here we discuss the lack of a need for state dependence when describing the directly reconstructable region.

In Appendix A, we analyze two-sided black holes within our construction and discuss how a version of complementarity works in this setup. Appendixes B and C collect explicit calculations for Schwarzschild-AdS and the de Sitter limit of FRW spacetimes, respectively.

\section{MAXIMALLY ENTROPIC STATES HAVE NO SPACETIME}

In this section, we see that maximally entangled states in holographic theories do not have directly reconstructable spacetime. First, we lay out the conditions for reconstructability in general theories of holographic spacetimes. Then we examine the familiar example of a large static black hole in AdS spacetime and determine its reconstructable region. We then discuss the de Sitter limit of flat FRW spacetimes. Finally, we prove a theorem establishing that maximally entropic holographic states have no reconstructable spacetime.

\section{A. Holographic reconstructability}

In order to argue that typical states have no reconstructable region, we must first present the conditions for a region of spacetime to be reconstructed from the boundary theory. We adopt the formalism presented first in Ref. [8] but appropriately generalized in Ref. [6] to theories living on holographic screens [18] (which naturally includes the boundary of AdS spacetime as in the AdS/CFT correspondence).

The question to answer is as follows: "Given a boundary state and its time evolution with a known gravitational bulk dual, what regions of the bulk can be reconstructed?" This may sound tautological, but it is not. Settings in which this question is nontrivial include spacetimes with black holes and other singularities. From entanglement wedge reconstruction $[19,20]$, we know that the information of a pure black hole is contained in the boundary theory but whether or not the interior is reconstructable is unknown. In holographic theories of general spacetimes, we are interested in describing spacetimes with big bang singularities and a natural question is whether or not the theory reconstructs spacetime arbitrarily close to the initial singularity.

To answer this question, Ref. [8] proposed that reconstructable points in a spacetime are precisely those that can be localized at the intersection of entanglement wedges. This is similar to the proposal in Ref. [21] which advocates that reconstructable points are those located at the intersection of HRRT surfaces anchored to arbitrary achronal subregions of the AdS conformal boundary. However, this construction lacks the ability to localize points in entanglement shadows, which can form in rather tame spacetimes (e.g. a neutron star in AdS spacetime), while using the intersection of entanglement wedges allows us to probe these regions.

In order to generalize this to theories living on holographic screens, an essential change is that one can only consider HRRT surfaces anchored to the leaves of a given holographic screen (usually associated to a fixed reference frame) [6]. This is because holographic screens have a unique foliation into leaves that corresponds to a particular time foliation of the holographic theory. Thus the von Neumann entropy of subregions in the holographic theory only makes sense for subregions of a single leaf. Note that despite the lack of a unique time foliation of the conformal boundary, this subtlety is also present in AdS/CFT. Namely, one should consider only a single time foliation of the boundary and the HRRT surfaces anchored to the associated equal time slices even in asymptotically AdS spacetimes [6]. ${ }^{1}$ This issue becomes manifest when the boundary contains multiple disconnected components, as we discuss in Appendix A.

Thus we define the reconstructable region of a spacetime as the union of all points that can be localized at the boundary of entanglement wedges of all subregions of leaves of the holographic screen. Henceforth, we refer to the regions of spacetime constructed in this way as the directly reconstructable regions (or simply the reconstructable regions when the context is clear), and our analysis primarily focuses on these regions. For a more detailed study of directly reconstructable regions in general spacetimes, see Ref. [6]. In particular, this definition only allows for the reconstruction of points outside the horizon for a quasistatic one-sided black hole, since such a horizon acts as an extremal surface barrier [23]. ${ }^{2}$ This also prevents the direct reconstruction of points near singularities such as big bang singularities and the black hole singularity of a two-sided black hole.

Now that we have detailed the conditions for regions of spacetime to be directly reconstructable, we must determine a measure of "how much" spacetime is reconstructable. This allows us to see the loss of spacetime in the limit of states becoming typical. In the context of quantum error correction [17], we are attempting to quantify the

\footnotetext{
${ }^{1}$ This is related to the work in Ref. [22], which studied the breakdown of the HRRT formula in certain limits of boundary subregions. These breakdowns correspond to disallowed foliations of the boundary theory.

${ }^{2}$ This does not exclude the possibility that the holographic theory allows for some effective description of regions other than the directly reconstructable one, e.g. the black hole interior (perhaps along the lines of Ref. [24]). This may make the interior spacetime manifest, perhaps at the cost of losing the local description elsewhere, and may be necessary to describe the fate of a physical object falling into a black hole. We focus on spacetime regions that can be described by the boundary theory without resorting to such descriptions.
} 
factorization of the code subspace, e.g. how many dangling bulk legs exist in a tensor network representation of the code $[25,26]$. We expect the spacetime volume of the reconstructable region to be indicative to this property, and we use it in our subsequent analyses. The bulk spacetime directly reconstructable from a single leaf depends on features of the bulk, for example, the existence of shadows and time dependence. In the case of $(d+1)$-dimensional flat FRW spacetimes, we find that a codimension- 0 region can be reconstructed from a single leaf. On the other hand, in any static spacetime, all HRRT surfaces anchored to one leaf live in the same time slice in the bulk, and hence their intersections reconstruct a codimension- 1 surface of the bulk. This is the case in an AdS black hole.

The discrepancy of the dimensions of the directly reconstructable regions for different spacetimes of interest may seem to cause issues when trying to compare the loss of spacetime in these systems. Namely, it seems difficult to compare the loss of reconstructable spacetime in Schwarzschild-AdS spacetime as we increase the black hole mass to the loss of spacetime in the $w \rightarrow-1$ limit of flat FRW spacetimes. However, in all cases, the spacetime region directly reconstructable from a small time interval in the boundary theory is codimension 0 . We can then examine the relative loss of spacetime in both cases (black hole horizon approaching the boundary in AdS spacetime and $w \rightarrow-1$ in FRW spacetimes) by taking the ratio of the volume of the reconstructable region to the reconstructable volume of some reference state (e.g. pure AdS spacetime and flat FRW spacetime with some fixed $w \neq-1$ ). In static spacetimes, this reduces to a ratio of the spatial volumes reconstructed on a codimension-1 slice, allowing us to consider only the volume of regions reconstructed from single leaves.

\section{B. Large AdS black holes}

Here we see how spacetime disappears as we increase the mass of the black hole in static Schwarzschild-AdS spacetime, making the corresponding holographic state maximally entangled. We consider a holographic pure state living on the (single) conformal boundary of AdS spacetime. We introduce an infrared cutoff $r \leq R$ in $\operatorname{AdS}$ spacetime and consider a $d+1$-dimensional large black hole with horizon radius $r=r_{+}$.

As discussed in Sec. II A, the size of the spacetime region directly reconstructable from the boundary theory is characterized by $V\left(r_{+}, R\right)$, the spatial volume between the black hole horizon and the cutoff. We normalize it by the volume of the region $r \leq R$ in empty AdS spacetime, $V(R)$, to get the ratio

$f\left(\frac{r_{+}}{R}\right) \equiv \frac{V\left(r_{+}, R\right)}{V(R)}=(d-1) \frac{r_{+}^{d-1}}{R^{d-1}} \int_{1}^{\frac{R}{r_{+}}} \frac{x^{d-2}}{\sqrt{1-\frac{1}{x^{d}}}} d x$,

which depends only on $r_{+} / R$ (and $d$ ). As expected, it behaves as

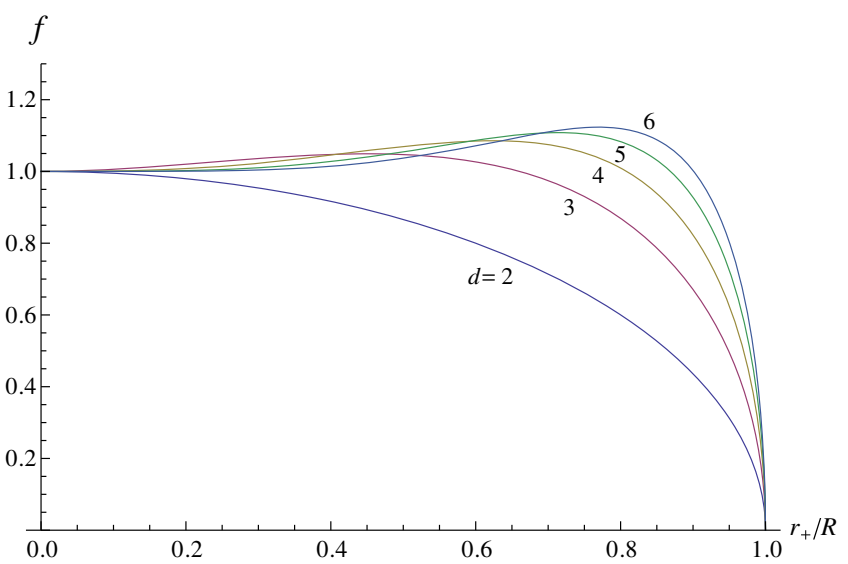

FIG. 1. The volume $V\left(r_{+}, R\right)$ of the Schwarzschild-AdS spacetime that can be reconstructed from the boundary theory, normalized by the corresponding volume $V(R)$ in empty AdS spacetime: $f=V\left(r_{+}, R\right) / V(R)$. Here, $R$ is the infrared cutoff of $(d+1)$-dimensional AdS spacetime, and $r_{+}$is the horizon radius of the black hole.

$$
f\left(\frac{r_{+}}{R}\right) \begin{cases}\simeq 1 & \left(r_{+} \ll R\right) \\ \rightarrow 0 & \left(r_{+} \rightarrow R\right)\end{cases}
$$

in the two opposite limits. The details of this calculation can be found in Appendix B 1. Here, we plot $f\left(r_{+} / R\right)$ in Fig. 1 for various values of $d$.

In the limit $r_{+} \rightarrow R$, the HRRT surface, $\gamma_{A}$, anchored to the boundary of subregion $A$ of a boundary space (a constant $t$ slice of the $r=R$ hypersurface) becomes the region $A$ itself or the complement, $\bar{A}$, of $A$ on the boundary space, whichever has the smaller volume. ${ }^{3}$ This implies that the entanglement entropy of $A$, given by the area of the HRRT surface as $S_{A}=\left\|\gamma_{A}\right\| / 4 l_{\mathrm{P}}^{d-1}$, becomes exactly proportional to the smaller of the volumes of $A$ and $\bar{A}$ in the boundary theory,

$$
S_{A}=\frac{1}{4 l_{\mathrm{P}}^{d-1}} \min \{\|A\|,\|\bar{A}\|\} .
$$

Here, $\|x\|$ represents the volume of the object $x$ (often called the area for a codimension-2 surface in spacetime), and $l_{\mathrm{P}}$ is the $(d+1)$-dimensional Planck length in the bulk. Via usual thermodynamic arguments, we interpret this to mean that the state in the boundary theory is generic, so that it obeys the Page law [16]. ${ }^{4}$ This in turn implies that the temperature of the system, which is

\footnotetext{
${ }^{3}$ We do not impose a homology constraint, since we consider a pure state in the holographic theory. Additionally, we only consider subregions larger than the cutoff size.

${ }^{4}$ Page's analysis tells us that for a generic state (a Haar random state) in a Hilbert space, the entanglement entropy of a reduced state is nearly maximal. In fact, at the level of the approximation we employ in this paper, $\|A\| / l_{\mathrm{P}}^{d-1} \rightarrow \infty$, such a state has the maximal entanglement entropy for any subregion, Eq. (3).
} 
identified as the Hawking temperature $T_{\mathrm{H}}$, is at the cutoff scale. ${ }^{5} T_{\mathrm{H}}$ is related to $r_{+}$by

$$
\frac{r_{+}}{R}=\frac{4 \pi l^{2}}{d R} T_{\mathrm{H}},
$$

where $l$ is the AdS radius. Hence, the cutoff scale of the boundary theory is given by [27]

$$
\Lambda=\frac{d R}{4 \pi l^{2}} .
$$

This allows us to interpret the horizontal axis of Fig. 1 as $T_{\mathrm{H}} / \Lambda$ from the viewpoint of the boundary theory.

We finally make a few comments. First, it is important to note that by the infrared cutoff, we do not mean that the spacetime literally ends there as in the scenario of Ref. [28]. Such termination of spacetime would introduce dynamical gravity in the holographic theory, making the maximum entropy of a subregion scale as the area, rather than the volume, in the holographic theory. Rather, our infrared cutoff here means that we focus only on the degrees of freedom in the bulk deeper than $r=R$, corresponding to setting the sliding renormalization scale to be $\approx R / l^{2}$ in the boundary theory. In particular, the boundary theory is still nongravitational.

Second, to state that spacetime disappears in the limit where the holographic state becomes typical, it is crucial to define spacetime as the directly reconstructable region. This becomes clear by considering a large subregion $A$ on the boundary theory such that $A$ and its HRRT surface $\gamma_{A}$ enclose the black hole at the center. If we take the simple viewpoint of entanglement wedge reconstruction, this would say that spacetime does not disappear even if the black hole becomes large and its horizon approaches the cutoff surface, since the black hole interior is within the entanglement wedge of $A$ so that it still exists in the sense of entanglement wedge reconstruction. We, however, claim that such a region does not exist as a localizable spacetime region, as explained in Sec. II A.

Third, the curves in Fig. 1 are not monotonically decreasing as $r_{+}$increases for $d>2$, despite the fact that

$$
\frac{d}{d r_{+}}\left\{S_{A, \max }-S_{A, \mathrm{BH}}\left(r_{+}\right)\right\}<0 .
$$

Here, $S_{A, \max }$ and $S_{A, \mathrm{BH}}\left(r_{+}\right)$are the maximal entropy and the entropy corresponding to the black hole geometry of subregion $A$, given by

$$
S_{A, \max }=\frac{\|A\|}{4 l_{\mathrm{P}}^{d-1}}, \quad S_{A, \mathrm{BH}}\left(r_{+}\right)=\frac{\|A\|}{4 l_{\mathrm{P}}^{d-1}} \frac{r_{+}^{d-1}}{R^{d-1}} .
$$

\footnotetext{
${ }^{5}$ When we refer to a high temperature state, we do not mean that the whole holographic state is a mixed thermal state. What we really mean is a high energy state, since we focus on pure states.
}

This increase in spacetime volume may be demonstrating that the additional entanglement in the boundary state allows for more bulk nodes in the code subspace. Alternatively, this may be a feature of using volume as our measure. Regardless, the decrease observed near the cutoff temperature is the main focus of our attention, and we expect any other reasonable measure to correspondingly vanish.

Finally, the statement that spacetime disappears as the holographic state approaches typicality persists for twosided black holes. In this setup, there is a new issue that does not exist in the case of single-sided black holes: the choice of a reference frame associated with a relative time shift between the two boundaries. The discussion of twosided black holes is given in Appendix A.

\section{C. de Sitter states are maximally entropic}

We have seen that a large black hole in AdS spacetime with $r_{+} \rightarrow R$ corresponds to CFT states at the cutoff temperature, and that the holographic states in this limit have the entanglement entropy structure of Eq. (3). Below, we refer to states exhibiting Eq. (3) as the maximally entropic states. Is there an analogous situation in the holographic theory of FRW spacetimes, described in Ref. [5]? Here we argue that the de Sitter limit $(w \rightarrow-1)$ in flat FRW universes provides one. ${ }^{6}$

We first see that the holographic state becomes maximally entropic in the case that a universe approaches de Sitter space at late times [15]. This situation arises when the universe contains multiple fluid components including one with $w=-1$, so that it is dominated by the $w=-1$ component at late times. This analysis does not apply directly to the case of a single component with $w=-1+\epsilon$ $\left(\epsilon \rightarrow 0^{+}\right)$, which is discussed later.

In the universe under consideration, the FRW metric approaches the de Sitter metric in flat slicing at late times

$$
d s^{2}=-d t^{2}+e^{\frac{2 t}{\alpha}}\left(d r^{2}+r^{2} d \Omega_{d-1}^{2}\right),
$$

where $\alpha$ is the Hubble radius, and we have taken the spacetime dimension of the bulk to be $d+1$. The Penrose diagram of this spacetime is depicted in Fig. 2, where constant time slices are drawn and the region covered by the coordinates is shaded; future timelike infinity $I_{+}$ corresponds to $t=\infty$, while the null hypersurface $N$ corresponds to $t=-\infty$. At late times, the past holographic screen of the FRW universe asymptotes to the codimension1 null hypersurface $\Sigma^{\prime}$ depicted in the figure. This hypersurface is located at

$$
r=\alpha e^{-\frac{t}{\alpha}}
$$

\footnotetext{
${ }^{6}$ For a simple proof applicable to $2+1$ dimensions, see Appendix C 1.
} 


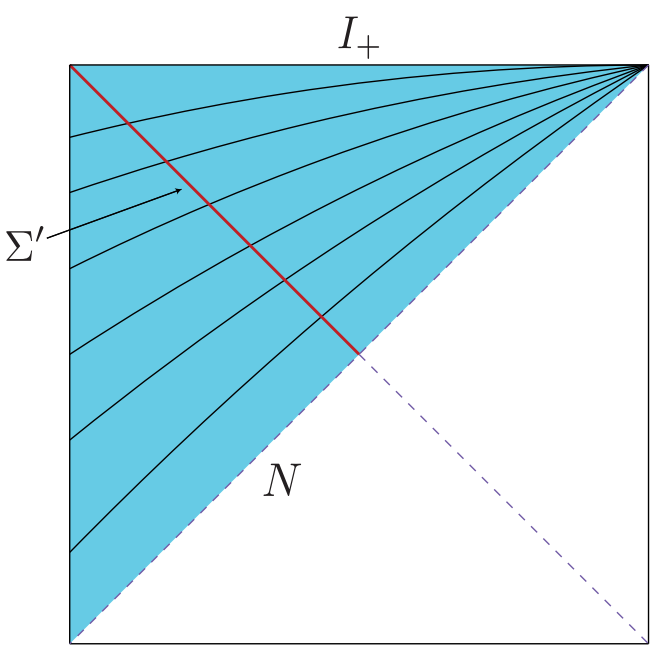

FIG. 2. The Penrose diagram of de Sitter space. The spacetime region covered by the flat-slicing coordinates is shaded, and constant time slices in this coordinate system are drawn. The codimension-1 null hypersurface $\Sigma^{\prime}$ is the cosmological horizon for an observer at $r=0$, to which the holographic screen of the FRW universe asymptotes in the future.

which corresponds to the cosmological horizon for an observer moving along the $r=0$ geodesic.

We can now transform the coordinates to static slicing

$$
d s^{2}=-\left(1-\frac{\rho^{2}}{\alpha^{2}}\right) d \tau^{2}+\frac{1}{1-\frac{\rho^{2}}{\alpha^{2}}} d \rho^{2}+\rho^{2} d \Omega_{d-1}^{2} .
$$

In Fig. 3, we depict constant $\tau$ (red) and constant $\rho$ (blue) slices, with the shaded region being covered by the coordinates. This metric makes it manifest that the spacetime has a Killing symmetry corresponding to $\tau$ translation. Using this symmetry, we can map a leaf of the original FRW universe to the $\tau=0$ hypersurface, $\Sigma$. Since the leaf of the universe under consideration approaches arbitrarily close to Eq. (9) at late times, the image of the map, $\Xi^{\prime}$, asymptotes to the bifurcation surface $\Xi$ at

$$
\rho=\alpha,
$$

for a leaf at later times.

Consider an arbitrary subregion $A$ on $\Xi^{\prime}$ and the minimal area surface $\gamma_{A}$ on $\Sigma$ anchored to the boundary of $A, \partial A$. Since the geometry of $\Sigma$ is $S^{d}$ with $\Xi$ being an equator, the minimal area surface $\gamma_{A}$ becomes the region $A$ itself (or its complement on $\Xi^{\prime}$, whichever is smaller) in the limit $\Xi^{\prime} \rightarrow \Xi$. Strictly speaking, this statement does not apply for a small subset of subregions, since $\Xi^{\prime}$ is not exactly $\Xi$ unless the leaf under consideration is at strictly infinite time. (For subregions in this subset, the minimal area surfaces probe $\rho \ll \alpha$. For spherical caps, these subregions are approximately hemispheres.) However, the fractional size of the subset goes to 0 as we focus on later leaves.

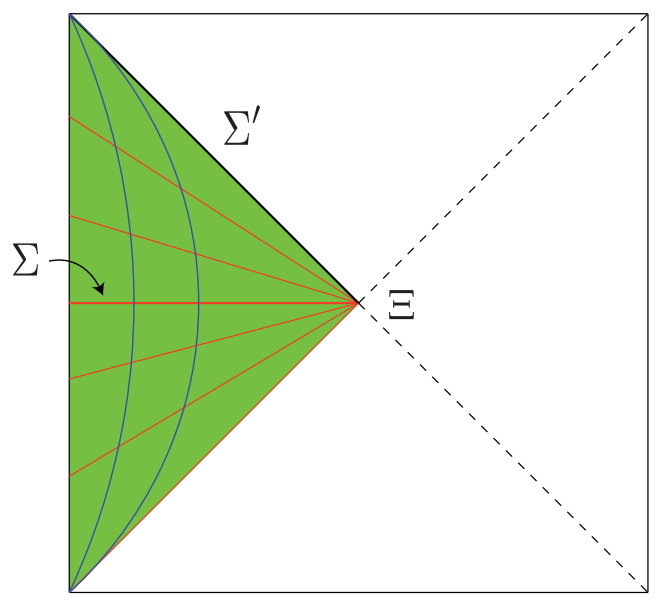

FIG. 3. Constant time slices and the spacetime region covered by the coordinates in static slicing of de Sitter space. Here, $\Sigma$ is the $\tau=0$ hypersurface, and $\Xi$ is the bifurcation surface, given by $\rho=\alpha$ with finite $\tau$.

Continuity then tells us that our conclusion persists for all subregions.

The surface $\gamma_{A}$ found above is in fact an extremal surface, since the bifurcation surface $\Xi$ is an extremal surface, so any subregion of it is also extremal. It is easy to show that this surface is indeed the HRRT surface, the minimal area extremal surface. Suppose there is another extremal surface $\gamma_{A}^{\prime}$ anchored to $\partial A$. We could then send a null congruence from $\gamma_{A}^{\prime}$ down to $\Sigma$, yielding another codimension-2 surface $\gamma_{A}^{\prime \prime}$ given by the intersection of the null congruence and $\Sigma$. Because $\gamma_{A}^{\prime}$ is extremal, the focusing of the null rays implies $\left\|\gamma_{A}^{\prime}\right\|>\left\|\gamma_{A}^{\prime \prime}\right\|$, and by construction $\left\|\gamma_{A}\right\|<\left\|\gamma_{A}^{\prime \prime}\right\|$. This implies that $\gamma_{A}$ is the HRRT surface, and hence

$$
S_{A}=\frac{1}{4 l_{\mathrm{P}}^{d-1}} \min \{\|A\|,\|\bar{A}\|\} .
$$

Namely, the holographic state representing a FRW universe that asymptotically approaches de Sitter space becomes a maximally entropic state in the late time limit.

The global spacetime structure in the case of a single fluid component with $w \neq-1$ is qualitatively different from the case discussed above. For example, the area of a leaf grows indefinitely. However, for any finite time interval, the behavior of the system approaches that of de Sitter space in the limit $w \rightarrow-1$. In fact, the numerical analysis of Ref. [5] tells us that the holographic entanglement entropy of a spherical cap region becomes maximal in the $w \rightarrow-1$ limit. We show in Appendix C 2 that this occurs for an arbitrary subregion on a leaf.

\section{Spacetime disappears as $w \rightarrow-1$ in the holographic FRW theory}

We have seen in our AdS/CFT example that as the holographic state approaches typicality, and hence becomes 
maximally entropic, the directly reconstructable region disappears. On the other hand, we have shown that the entanglement entropies for flat FRW universes approaches the maximal form as $w \rightarrow-1$. Does this limit have a corresponding disappearance of reconstructable spacetime? Here we show that the answer to this question is yes.

From the analysis of Sec. II C, we see that a leaf at late times in universes approaching de Sitter space can be mapped to a surface on the $\tau=0$ hypersurface $\Sigma$, which asymptotes to the bifurcation surface $\Xi$ in the late time limit. From the Killing symmetry, the HRRT surfaces anchored to this mapped leaf must all be restricted to living on $\Sigma$. Mapping the HRRT surfaces back to the original location, we see that they asymptote to living on the null hypersurface $\Sigma^{\prime}$. Thus, we find that the HRRT surface for any subregion of a leaf $\sigma_{*}$ asymptotes to the future boundary of the causal region $D_{\sigma_{*}}$, which we denote by $\partial D_{\sigma_{*}}^{(+)}$, as a universe approaches de Sitter space. A similar argument holds for universes where $w \rightarrow-1$. In Appendix C 3, we present some examples where we can see this behavior using analytic expressions for HRRT surfaces.

What does this imply for the reconstructable region in de Sitter space? Using the prescription outlined in Sec. II A, we find that spacetime points on the future causal boundary of a leaf, $\partial D_{\sigma_{*}}^{(+)}$, can be reconstructed. This is a codimension-1 region in spacetime. One might then think that we can reconstruct a codimension- 0 region by considering multiple leaves, as was the case in a Schwarzschild-AdS black hole. However, the holographic screen of de Sitter space is itself a null hypersurface, with future leaves lying precisely on the future causal boundary of past leaves. This means that even by using multiple leaves we cannot reconstruct any nonzero measure spacetime region in the de Sitter (and $w \rightarrow-1$ ) limit.

We now compute the reconstructable region in $(2+1)$ dimensional flat FRW spacetimes. As discussed in Sec. II A, this region is comprised of points that can be localized as the intersection of edges of entanglement wedges. We consider the reconstructable region associated to a single leaf, and hence this prescription reduces to finding points located at the intersection of HRRT surfaces anchored to the leaf. This alone gives us a codimension- 0 reconstructable region. In $(2+1)$-dimensional FRW spacetimes, HRRT surfaces are simply geodesics in the bulk spacetime, and this problem becomes tractable.

For a $(2+1)$-dimensional flat FRW universe filled with a single fluid component $w$, the leaf of the holographic screen at conformal time $\eta_{*}$ is located at coordinate radius

$$
r_{*}=\left.\frac{a}{\dot{a}}\right|_{\eta=\eta_{*}}=w \eta_{*}
$$

Let us parametrize the points on the leaf by $\phi \in[0,2 \pi)$. Consider an interval of the leaf at time $\eta_{*}$ centered at $\phi_{0}$ with half opening angle $\psi$. The HRRT surface of this subregion is simply the geodesic connecting the end points of the interval: $(\eta, \phi)=\left(\eta_{*}, \phi_{0}-\psi\right)$ and $\left(\eta_{*}, \phi_{0}+\psi\right)$. It is clear from the symmetry of the setup that if we consider a second geodesic anchored to an interval with the same opening angle but with a center $\phi_{0}^{\prime} \in\left[\phi_{0}-2 \psi, \phi_{0}+2 \psi\right]$, then the two geodesics intersect at a point, specifically where $\phi=\left(\phi_{0}+\phi_{0}^{\prime}\right) / 2$. Using these pairs of geodesics, it is clear that we can reconstruct all points on all geodesics anchored to the leaf. The union of these points gives us a codimension-0 region.

Can we get a larger region? In $(2+1)$-dimensional flat FRW spacetimes, the answer is no. In higher dimensions, knowing the HRRT surfaces for all spherical cap regions may not be sufficient to figure out reconstructable regions; for example, one may consider using disjoint regions in the hopes that the new HRRT surfaces would explore regions inaccessible to the previous HRRT surfaces (although we do not know if this really leads to a larger reconstructable region). However, in $2+1$ dimensions, both connected and disconnected phases of extremal surfaces are constructed from the geodesics already considered, so we gain nothing from considering disconnected subregions. We thus find that the set of all points on HRRT surfaces anchored to arbitrary subregions on a leaf is exactly the reconstructable region from the state on the leaf.

In Fig. 4, we show a plot of the reconstructable spacetime volume as a function of $w$. It shows a qualitatively similar behavior to that of Fig. 1, where the reconstructable volume increases and then sharply declines to 0 as the holographic state becomes maximally entropic.

We can also perform a similar analysis in higher dimensions. Due to the numerical difficulty in finding extremal surfaces, here we restrict ourselves to the region reconstructable by spherical cap regions (which may indeed be the fully reconstructable region) and to only a few representative values of $w$. The results are plotted in Fig. 5

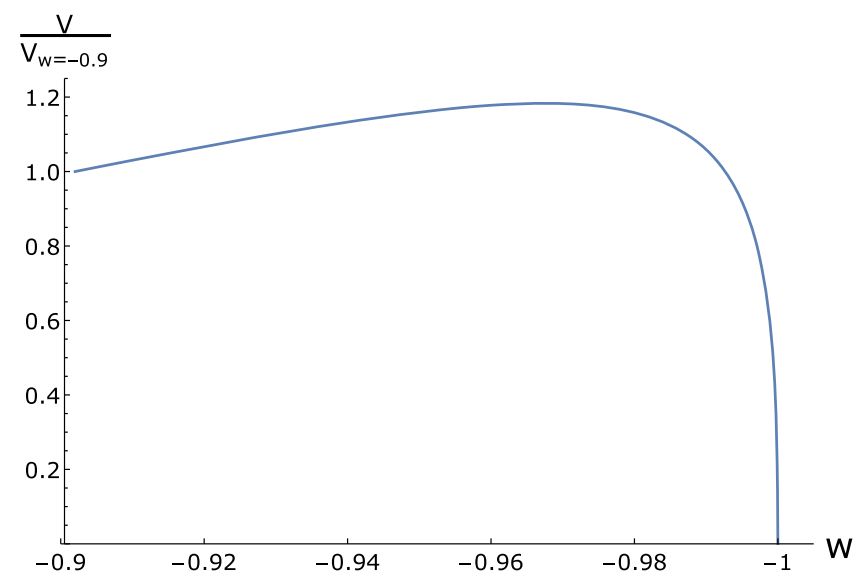

FIG. 4. The spacetime volume of the reconstructable region in $(2+1)$-dimensional flat FRW universes for $w \in(-0.9,-1)$, normalized by the reconstructable volume for $w=-0.9$. 


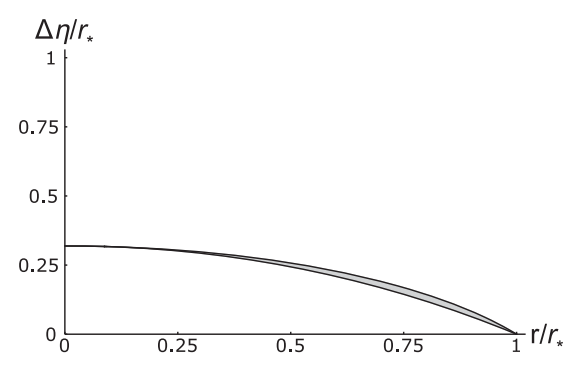

(a) $w=1, \eta_{*}=1$

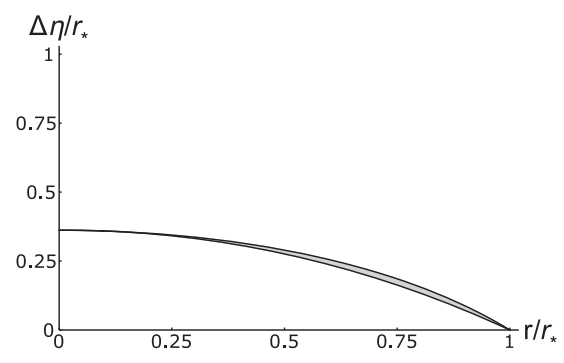

(b) $w=0, \eta_{*}=1$

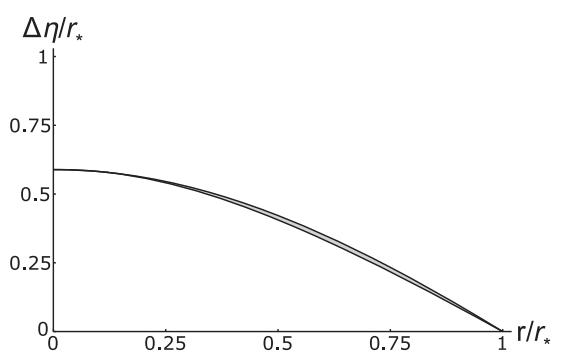

(c) $w=-4 / 5, \eta_{*}=-1$

FIG. 5. reconstructable spacetime regions for various values of $w$ in $(3+1)$-dimensional flat FRW universes. The horizontal axis is the distance from the center, normalized by that to the leaf. The vertical axis is the difference in conformal time from the leaf, normalized such that the null ray from the leaf would reach 1 . The full reconstructable region for each leaf would be the gray region between the two lines rotated about the vertical axis.

for $(3+1)$-dimensional FRW universes. These demonstrate the behavior that the extremal surfaces, and hence the reconstructable region, become more and more null as $w \rightarrow-1$.

The discussion in this subsection says that the reconstructable spacetime region disappears in the holographic theory of FRW spacetimes as the holographic state becomes maximally entropic in the de Sitter limit. While a microstate becoming maximally entropic does not directly mean that states representing the corresponding spacetime become typical in the holographic Hilbert space (since the number of independent microstates could still be small), we expect that the former indeed implies the latter as usual thermodynamic intuition suggests; see Sec. IV for further discussion. In any event, since typical states in a holographic theory are maximally entropic, we expect that the reconstructable spacetime region disappears as the holographic state becomes typical.

An important implication of the analysis here is that a holographic theory of de Sitter space cannot be obtained by taking a limit in the holographic theory of FRW spacetimes. A holographic theory of exact de Sitter space, if any, would have to be formulated in a different manner. ${ }^{7}$

\section{E. Maximally entropic states have no spacetime}

In this subsection, we provide a proof for the statement that the directly reconstructable region of a maximally entropic leaf is either the leaf itself or a subset of its null

\footnotetext{
${ }^{7}$ Another instance in which spacetime disappears is when the holographic description changes from that based on a past holographic screen (foliated by marginally antitrapped surfaces) to a future holographic screen (marginally trapped surfaces). Such a change of description may occur in a spacetime with a late time collapsing phase, e.g. in a closed FRW universe with the holographic screen constructed naturally in an observer-centric manner. (For an interpretation of such spacetime, see Ref. [5].) Since the leaf at the time of the transition is extremal, the analysis here indicates that the spacetime region reconstructable from a single leaf disappears at that time. This makes the necessity of the change of the description more natural.
}

cone. We use this result to argue that maximally entropic states have no spacetime. This heavily utilizes the maximin techniques developed in Ref. [29].

Theorem 1: Consider a compact codimension-2 spacelike surface, $\sigma$, with area $\mathcal{A}$, living in a spacetime that satisfies $R_{a b} v^{a} v^{b} \geq 0$ for all null vectors $v^{a}$. Suppose HRRT surfaces can consistently be anchored to $\sigma .^{8}$ Let $m(\Gamma)$ denote the HRRT surface anchored to the boundary, $\partial \Gamma$, of a subregion $\Gamma$ of $\sigma$.

If $\|m(\Gamma)\|=\min \{\|\Gamma\|,\|\bar{\Gamma}\|\}, \forall \Gamma \subset \sigma$, then either $\sigma$ is a bifurcation surface or all of the HRRT surfaces of $\sigma$ lie on a nonexpanding null hypersurface connected to $\sigma$.

Proof.-If $\Gamma_{1}$ and $\Gamma_{2}$ are subregions of $\sigma$, we abbreviate $\Gamma_{1} \cup \Gamma_{2}$ as $\Gamma_{1} \Gamma_{2}$. Let $m(\Gamma)_{\Sigma}$ denote the representative of $m(\Gamma)$ on a complete achronal surface $\Sigma$, defined by the intersection of $\Sigma$ with a null congruence shot out from $m(\Gamma)$. From the extremality of $m(\Gamma), R_{a b} v^{a} v^{b} \geq 0$, and the Raychaudhuri equation, $\left\|m(\Gamma)_{\Sigma}\right\| \leq\|m(\Gamma)\|$.

Consider three connected subregions $A, B, C$ of $\sigma$ such that $\partial A \cap \partial B \neq \varnothing, \partial B \cap \partial C \neq \varnothing$ where both such intersections are codimension 3, and $\|A \cup B \cup C\| \leq\|\sigma\| / 2$; see Fig. 6 for a diagram. By Theorem 17.h of Ref. [29], take $m(A B C)$ and $m(B)$ to be on the same achronal surface, $\Sigma$. Now, consider the representatives $m(A B)_{\Sigma}$ and $m(B C)_{\Sigma}$. From the properties of representatives and maximin surfaces, we have

$$
\begin{aligned}
S(A B)+S(B C) & \geq \frac{\left\|m(A B)_{\Sigma}\right\|}{4 l_{\mathrm{P}}^{d-1}}+\frac{\left\|m(B C)_{\Sigma}\right\|}{4 l_{\mathrm{P}}^{d-1}} \\
& \geq S(A B C)+S(B) .
\end{aligned}
$$

The assumption of maximal entropies then tells us that strong subadditivity is saturated, and hence

\footnotetext{
${ }^{8}$ This requires the expansion of the two null hypersurfaces bounding $D(\sigma)$ to have $\theta \leq 0$, where $D(\sigma)$ is the interior domain of dependence of some achronal set whose boundary is $\sigma$. These HRRT surfaces are guaranteed to exist and satisfy basic entanglement inequalities; see Refs. [15,30].
} 


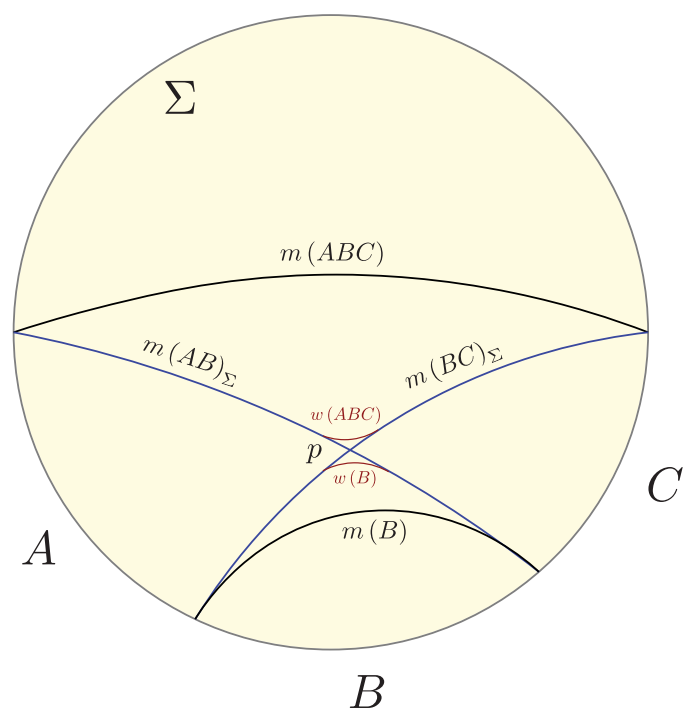

FIG. 6. Diagrams representing the achronal surface $\Sigma$ in which two HRRT surfaces, $m(A B C)$ and $m(B)$, live. $m(A B)_{\Sigma}$ and $m(B C)_{\Sigma}$ are the representatives of $m(A B)$ and $m(B C)$, respectively. They are shown to be intersecting at $p$. On a spacelike $\Sigma$, one could deform around this intersection to create two new surfaces with smaller areas.

$$
\begin{aligned}
\left\|m(A B)_{\Sigma}\right\| & =\|m(A B)\|, \\
\left\|m(B C)_{\Sigma}\right\| & =\|m(B C)\| .
\end{aligned}
$$

Additionally, $m(A B)_{\Sigma} \cap m(B C)_{\Sigma} \neq \varnothing$.

We have two cases depending on the nature of $\Sigma$.

Case 1: $m(A B C), m(B), m(A B)_{\Sigma}$, and $m(B C)_{\Sigma}$ live on $\Sigma$ which is a non-null hypersurface.

Suppose $m(A B)_{\Sigma} \cap m(B C)_{\Sigma}$ is a codimension-3 surface, meaning they intersect through some surface, $p$, depicted in Fig. 6. One could then smooth out the "corners" around $p$ to create new surfaces homologous to $A B C$ and $B$. This is depicted through the maroon lines in Fig. 6. By the triangle inequality, these new, smoothed out surfaces, $w(A B C)$ and $w(B)$, would have less total area than $m(A B C) \cup m(B)$ because $p \in \Sigma$, which is spacelike. However, this contradicts the minimality of $m(A B C)$ and $m(B)$,

$$
\begin{aligned}
& (\|A\|+\|B\|+\|C\|)+\|B\| \\
& \quad=\|m(A B C)\|+\|m(B)\| \\
& \quad \leq\|w(A B C)\|+\|w(B)\| \\
& \quad<\left\|m(A B)_{\Sigma}\right\|+\left\|m(B C)_{\Sigma}\right\| \\
& \quad=(\|A\|+\|B\|)+(\|B\|+\|C\|) .
\end{aligned}
$$

Therefore, $m(A B)_{\Sigma}$ and $m(B C)_{\Sigma}$ cannot intersect through some codimension-3 surface, yet they must still intersect. This requires $m(A B)_{\Sigma}$ and $m(B C)_{\Sigma}$ to coincide somewhere, a neighborhood of $x$, and by theorem 4.e of Ref. [29] these two surfaces must coincide at every point connected to $x$. This means that
$m(A B)=m(A) \cup m(B)$ and $m(B C)=m(B) \cup m(C)$. The only way this can consistently occur for all possible $A, B$, and $C$ is for $m(\Gamma) \subset \sigma$. This means that $\sigma$ itself is extremal, and hence is a bifurcation surface.

Case 2: $m(A B C), m(B), m(A B)_{\Sigma}$, and $m(B C)_{\Sigma}$ live on hypersurface $\Sigma$ which is at least partially null.

Suppose $m(A B)_{\Sigma} \cap m(B C)_{\Sigma}$ is a codimension-3 surface, $p$. If at $p, \Sigma$ is null and nonexpanding, then smoothing out the intersection does not result in new surfaces with smaller area. This is the condition that $\theta_{u}=0$ on the null hypersurface, $\Sigma_{u}$, coincident with $\Sigma$ at $p$, where $u$ is the null vector generating $\Sigma_{u}$ at $p$. Hence, the representatives can intersect at $p$ and simultaneously saturate strong subadditivity. Additionally, because $\left\|m(B C)_{\Sigma}\right\|=\|m(B C)\|$, we know that $\theta_{v}=0$ along the hypersurface generating the representatives of $m(B C)$, where $v$ is the null vector generating this hypersurface. Therefore at $p, \theta_{u}=\theta_{v}=0$.

We can now scan across $m(B C)_{\Sigma}$ by considering its intersection with $m\left(A B^{\prime}\right)_{\Sigma}$ where $\left(B^{\prime}, C^{\prime}\right)$ is a bipartition of $B \cup C$ where $\partial B^{\prime} \cap \partial A \neq \varnothing$, and then considering all such bipartitions. This is illustrated in Fig. 7 by splitting up $B \cup C$ at a few points labeled by $x_{i}$; for example, $B^{\prime}=\left[b, x_{3}\right]$ and $C^{\prime}=\left[x_{3}, c\right]$ is one such allowable bipartition. By continuity, all of $m(B C)_{\Sigma}$ is scanned. ${ }^{9}$

By the argument in the previous paragraph, all intersection points along $m(B C)_{\Sigma}$ must then have $\theta_{u}=\theta_{v}=0$. Assuming nondegeneracy, $m(B C)_{\Sigma}$ must therefore be the HRRT surface $m(B C)$. Additionally, every point of $m(B C)$ lives on some null, nonexpanding hypersurface and at $\partial m(B C)$ this surface connects to $\sigma$. Hence, at $\partial m(B C), \sigma$ must be marginal. This argument can be repeated for any set of appropriate subregions. This tells us that all HRRT surfaces have the previously stated properties.

Now, by theorem 17.h of Ref. [29], we can construct an achronal surface, $\Sigma$, that is foliated by HRRT surfaces. Each point of $\Sigma$ must now be null and nonexpanding. Additionally, the boundary of $\Sigma, \sigma$, must be marginal. Let $k$ denote the vector in this local marginal direction. This uniquely specifies $\Sigma$ as the null nonexpanding hypersurface generated by $k$. This is true for all $\Sigma$ foliated by HRRT surfaces, and each HRRT surface can belong to some foliation of a $\Sigma .{ }^{10}$ Hence all extremal surfaces anchored to $\sigma$ must belong to a nonexpanding null hypersurface.

\footnotetext{
${ }^{9}$ We believe this is sufficient to scan over the whole surface assuming the spacetime is smooth. Additionally, Eq. (15) requires there to be no energy density between a HRRT surface and its representative; this precludes jumps in the representatives due to entanglement shadows and the like.

${ }^{10}$ Under the assumption of the theorem, the HRRT surface of disconnected subregions is always disconnected. This is because the disconnected surface is extremal.
} 


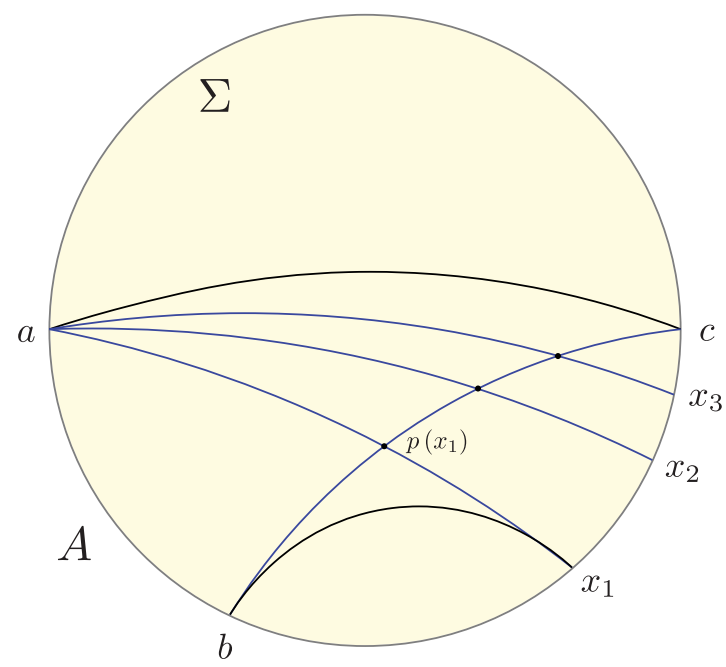

FIG. 7. This depicts how one can scan across the representative $m(B C)_{\Sigma}$ by bipartitioning $B C$ on the achronal surface $\Sigma$. At each of these intersections, $p\left(x_{i}\right), \theta_{u}=\theta_{v}=0$ if the state on the leaf is maximally entropic and $\Sigma$ is null and nonexpanding.

Back to the beginning, if the intersection of $m(A B)_{\Sigma}$ and $m(B C)_{\Sigma}$ is codimension 2, then the argument from case 1 applies and $\sigma$ must be extremal.

This concludes the proof of theorem 1 .

Corollary 1: Consider a codimension-2 surface, $\sigma$, with area $\mathcal{A}$, living in a spacetime satisfying $R_{a b} v^{a} v^{b} \geq 0$. Let $m(\Gamma)$ denote the HRRT surface anchored to $\partial \Gamma$.

If $\sigma$ is not marginal, then it cannot satisfy $\|m(\Gamma)\|=$ $\min \{\|\Gamma\|,\|\bar{\Gamma}\|\}, \forall \Gamma \subset \sigma$.

Proof.-The contrapositive of this statement is proven by theorem 1.

Consider the case that $\sigma$ is a leaf of a past holographic screen. If the leaf is extremal and the screen is not null, then the directly reconstructable spacetime is just the leaf itself. Additionally, this tells us the holographic screen must halt at this point. This indicates the end of a holographic description based on the past holographic screen. At this point, one can stitch the beginning of a new future holographic screen that starts at a bifurcation surface, patching together two holographic descriptions. This occurs in collapsing universes; see footnote 7 .

In the other case, if all of the HRRT surfaces of $\sigma$ have area corresponding to the maximal entropy, then all of the extremal surfaces must lie on the future null cone of the leaf, where this null cone is nonexpanding and compact. This cone itself is the limit of a past holographic screen because $\theta_{k}=0$. Barring the existence of a continuum of compact, nonexpanding, null hypersurfaces, the holographic screen then follows along this null surface from the leaf. Hence the directly reconstructable region will only be the screen itself, exactly as we observed in the case of de Sitter space. Again, we see that maximal entanglement corresponds to the end of a holographic description, but in this case the screen does not end; this corresponds to a stable final state.
In Sec. II B, we took the boundary to be at some large, fixed radius in AdS spacetime. One may be concerned that this cutoff surface is not marginal, and hence theorem 1 does not apply. However, in the limit that the black hole radius approaches the boundary, the statement holds because the horizon of the black hole satisfies the needed properties. Note that until this final limit, corollary 1 tells us that the entanglement of the boundary cannot be maximal.

Finally we are prepared to make a statement about typicality. Typical boundary states are maximally entangled, and hence the argument shows us that for holographic theories living on screens (an instance of which is AdS/CFT), typical states have no directly reconstructable spacetime.

\section{SPACETIME EMERGES THROUGH DEVIATIONS FROM MAXIMAL ENTROPY}

We have seen that when the holographic state becomes maximally entropic, spacetime defined as the directly reconstructable region disappears. Conversely, bulk spacetime emerges when we change parameters, e.g. the mass of the black hole or the equation of state parameter $w$, deviating the state from maximal entropy. In this section, we study how this deviation may occur and find qualitative differences between the cases of Schwarzschild-AdS and flat FRW spacetimes. This has important implications for the structures of holographic theories representing these spacetimes.

\section{A. CFT with subcutoff temperatures}

Consider the setup discussed in Sec. II B: a large black hole in asymptotically AdS spacetime. The holographic theory is then a local quantum (conformal) field theory. When the temperature of the system is at the cutoff scale, the holographic state has maximal entropies, Eq. (3). As we lower the temperature, the state deviates from a maximally entropic one, and correspondingly bulk spacetime emerges - the horizon of the black hole recedes from the cutoff surface, and the reconstructable spacetime region appears; see Fig. 1.

Suppose the temperature of the system $T$ is lower than the cutoff scale, $T<\Lambda$. We are interested in the behavior of von Neumann entropies of subregions of characteristic length $L$ in the boundary theory. These entropies are calculated holographically by finding the areas of the HRRT surfaces anchored to subregions of the cutoff surface $r=R$. We analyze this problem analytically for spherical cap regions in Appendix B 2. For sufficiently high temperature, $T \gg\left(\Lambda^{d-2} / l\right)^{1 /(d-1)}$, we find that the entanglement entropy for a subregion $A$ behaves as

$S_{A} \approx \begin{cases}c A_{d-2} L^{d-2} \Lambda^{d-2} & \text { for } L \ll L_{*}, \\ c A_{d-2} \frac{r_{+}^{d-1} L^{d-1}}{l^{2-2}} \approx c\left(\frac{T}{\Lambda}\right)^{d-1} A_{d-2} L^{d-1} \Lambda^{d-1} & \text { for } L \gg L_{*} .\end{cases}$ 
Here,

$$
L_{*} \approx \frac{l^{2} R^{d-2}}{r_{+}^{d-1}} \approx \frac{\Lambda^{d-2}}{T^{d-1}}
$$

$c \approx\left(l / l_{\mathrm{P}}\right)^{d-1}$ is the central charge of the CFT, and $A_{d-2}$ is the area of the $(d-2)$-dimensional unit sphere. We find that the scaling of the entanglement entropy changes (smoothly) from an area law to a volume law as $L$ increases. For $T \ll\left(\Lambda^{d-2} / l\right)^{1 /(d-1)}$, i.e. $L_{*} \gg l$, the entanglement entropy obeys an area law for all subregions. We note that the length in the boundary theory is still measured in terms of the $d$-dimensional metric at infinity with the conformal factor stripped off. The cutoff length is thus $1 / \Lambda \approx O\left(l^{2} / R\right)$, and the size of the boundary space is $\approx O(l)$.

While we have analyzed spherical cap subregions, the behavior of the entanglement entropy found above is more general. When the temperature is lowered from the cutoff scale, the entanglement entropy $S_{A}$ deviates from the maximal value. Defining

$$
Q_{A}=\frac{S_{A}}{S_{A, \max }}=\frac{S_{A}}{\|A\| / 4 l_{\mathrm{P}}^{d-1}},
$$

we find that

$$
Q_{A} \approx \begin{cases}\frac{1}{L \Lambda} & \text { for } L \ll \frac{\Lambda^{d-2}}{T^{d-1}}, \\ \left(\frac{T}{\Lambda}\right)^{d-1} & \text { for } L \gg \frac{\Lambda^{d-2}}{T^{d-1}} .\end{cases}
$$

Here, we have assumed that subregion $A$ is characterized by a single length scale $L$, and that the temperature is sufficiently high, $T \gg\left(\Lambda^{d-2} / l\right)^{1 /(d-1)}$. [If $T \ll\left(\Lambda^{d-2} / l\right)^{1 /(d-1)}, Q_{A} \approx$ $1 / L \Lambda$ for all subregions.] This behavior is depicted schematically in Fig. 8.

We find that as the temperature is lowered from the cutoff scale, two things occur for entanglement entropies.

(i) For sufficiently large subregions, the entanglement entropies still obey a volume law, but the coefficient becomes smaller.

(ii) The more the temperature is lowered, the further subregions have entanglement entropies obeying an area law. This occurs from shorter scales, i.e. subregions with smaller sizes.

These make the entanglement entropies deviate from the maximal value and lead to the emergence of reconstructable spacetime: the region between the black hole horizon and the cutoff surface, $r_{+}<r \leq R$.

\section{B. FRW universes with $w>-1$}

As spacetime emerges by reducing the mass of the black hole in the Schwarzschild-AdS case, a codimension-0 spacetime region that is reconstructable from a single leaf appears when $w$ is increased from -1 . As in the AdS case,

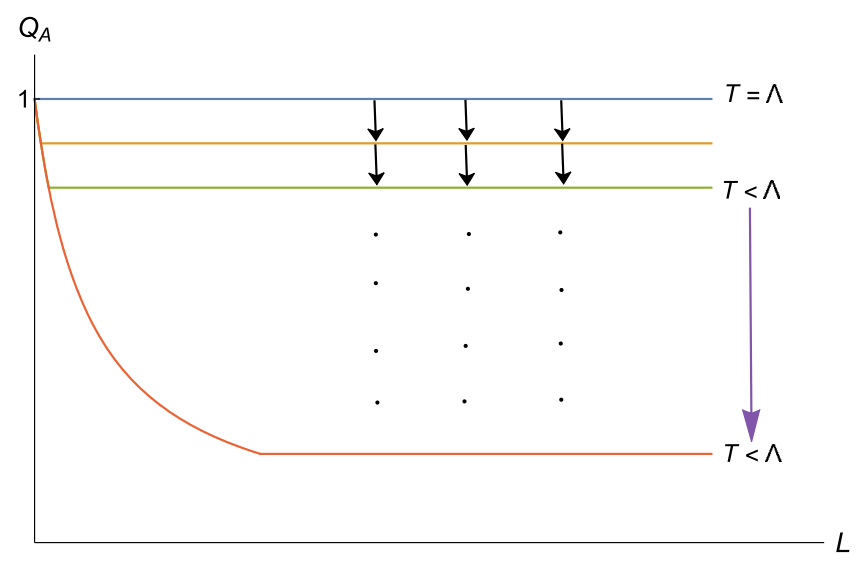

FIG. 8. A schematic depiction of the entanglement entropy in the Schwarzschild-AdS spacetime, normalized by the maximal value of entropy in the subregion, $Q_{A}=S_{A} / S_{A, \max }$, and depicted as a function of the size $L$ of subregion $A$; see Eq. (20). The scales of the axes are arbitrary. As the mass of the black hole is lowered (the temperature $T$ of the holographic theory is reduced from the cutoff $\Lambda), Q_{A}$ deviates from 1 in a specific manner.

this appearance is associated with a deviation of entanglement entropies from saturation. However, the manner in which this deviation occurs is qualitatively different in the two cases.

To illustrate the salient points, let us consider flat FRW spacetimes with a single fluid component $w$ and a spherical cap region $A$ on a leaf parametrized by the half opening angle $\psi$. Below, we focus on entanglement entropies $S_{w}(\psi)$ of the regions with $\psi \leq \pi / 2$. Those with $\psi>\pi / 2$ are given by the relation $S_{w}(\psi)=S_{w}(\pi-\psi)$.

As before, we define

$$
Q_{w}(\psi)=\frac{S_{w}(\psi)}{S_{\max }(\psi)}=\frac{S_{w}(\psi)}{\|A\| / 4 l_{\mathrm{P}}^{d-1}} .
$$

This quantity was calculated in Ref. [5] in $3+1$ dimensions, which we reproduce in Fig. 9. The basic features are similar in other dimensions. In particular, $Q_{w}(\psi)$ satisfies the properties given in Eqs. (C3) and (C4) in Appendix C 1.

We find that the way $Q_{w}(\psi)$ deviates from 1 as $w$ is increased from -1 is qualitatively different from the way the similar quantity $Q_{A}$ deviates from 1 in the SchwarzschildAdS case as the temperature is reduced from the cutoff scale. In particular, we find that in the FRW case

(i) the deviation from $Q_{w}(\psi)=1$ occurs from larger subregions. Namely, as $w$ is raised from $-1, Q_{w}(\psi)$ is reduced from 1 first in the vicinity of $\psi=\pi / 2$.

(ii) There is no regime in which the entanglement entropy obeys an area law, $Q_{w}(\psi) \sim 1 / \psi$, or a volume law with a reduced coefficient, $Q_{w}(\psi)=$ const $<1$.

As we see next, these have profound implications for the nature of the holographic theory of FRW spacetimes. 


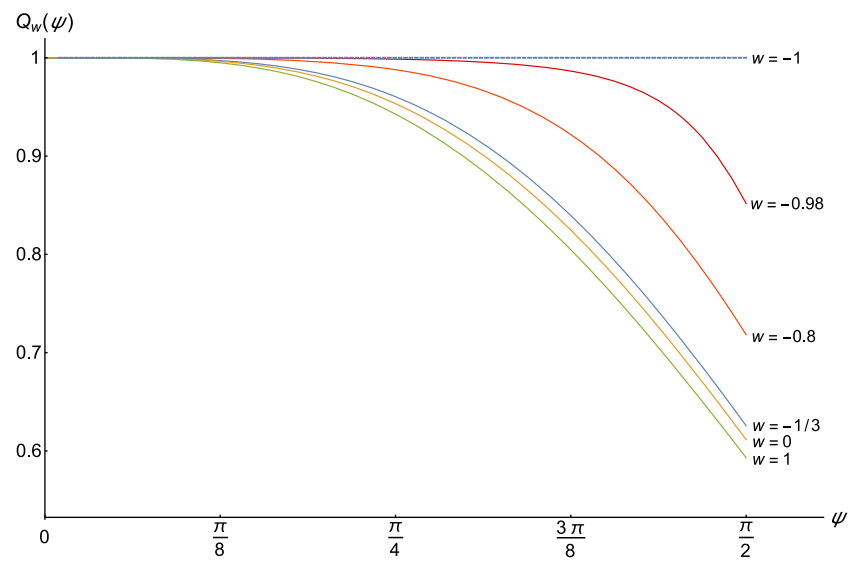

FIG. 9. The entanglement entropy in the holographic theory of flat FRW spacetimes normalized by the maximal value of entropy in the subregion, $Q_{w}(\psi)=S_{w}(\psi) / S_{\max }(\psi)$, as a function of the size of the subregion, a half opening angle $\psi$. As the equation of state parameter $w$ is increased from $-1, Q_{w}(\psi)$ deviates from 1 in a way different from the Schwarzschild-AdS case.

\section{Locality vs nonlocality}

In the following discussion, we assume that the dynamics in the holographic theory are chaotic and nonintegrable as expected in a theory of quantum gravity; see, e.g. Ref. [31]. Such systems are expected to satisfy the eigenstate thermalization hypothesis (ETH) [32,33], so generic high energy eigenstates reproduce the behavior of a thermal Gibbs density matrix. In addition, we note that the dimension of the holographic Hilbert space is large $\left(\mathcal{A} / 4 l_{\mathrm{P}}^{d-1} \gg 1\right)$ and finite size effects causing deviations from the thermodynamic limit can be ignored.

We have already seen that one way to obtain a maximally entropic state is to look at high energy states in a local theory. In the context of AdS/CFT, this corresponds to examining black holes with temperature near the cutoff scale. To deviate from maximal entropy, one can then simply lower the energy of the states being considered. For subregions beyond the correlation length, the reduced density matrix is well approximated by a Gibbs density matrix, and hence the entropy obeys a volume law but with a prefactor dependent on the temperature $T$. For length scales below the correlation length, the von Neumann entropy is dominated by the area law contribution. Together, these combine to give entanglement entropy curves that have the qualitative behavior shown in Fig. 8. Note that in a local theory, lowering the temperature shows deviation from thermal behavior originating at small length scales. Namely, the slope of $Q_{A}$ begins deviating from 0 at small scales. This entropy deviation at small scales is expected to be a general phenomenon of equilibrium states governed by a local Hamiltonian.

However, the entanglement entropy curves calculated for holographically FRW universes show drastically different behavior; see Fig. 9. ${ }^{11}$ Namely, the deviations from maximal entropy originate at large length scales, and the entanglement entropy for small subregions is maximal regardless of the fluid parameter $w$. Additionally, these entropy curves are invariant under time translation. This behavior cannot be achieved by a local theory. One may think that a Lifshitz-type theory with large $z$ may be able to accommodate such behavior due to large momentum coupling, but the leading order contribution to the entanglement entropy in $d$ dimensions is believed to be proportional to $(L / \epsilon)^{d-1-1 / z}$ for weakly coupled theories [34], where $L$ is the characteristic length of the entangling region and $\epsilon$ is the cutoff length. Thus entanglement entropy is proportional to the volume only in the limit that $z \rightarrow \infty$, which would be a nonlocal field theory. Indeed, entanglement entropy being maximal for small subregions is observed in a number of nonlocal theories [35-40] and is likely a generic phenomenon in such theories.

This leads us to believe that an appropriate holographic description of FRW universes would be nonlocal. ${ }^{12}$ This provides us with a few possibilities of theories that have the desired qualitative features, all of which have a freedom to tune a parameter which corresponds to changing $w$ (and hence the entropy).

(I) A nonlocal theory with a characteristic length scale below the system size, changing the nonlocal length scale of the theory or energy of the state.

(II) A nonlocal theory coupling sites together at all length scales (like a long-range interacting spin chain or a variant of the Sachdev-Ye-Kitaev (SYK) model [41-43] with all-to-all random coupling between a fixed number, $q$, of sites, $\mathrm{SYK}_{q}$ ), changing the energy of the state.

(III) A nonlocal theory with a fundamental parameter controlling the coupling at all scales in which variations can change the entropy, for example, changing the number of sites coupled to each other in each term of the Hamiltonian (analogous to changing $q$ in $\mathrm{SYK}_{q}$ ).

The ground states of theories in case (I) are explored in Refs. [35-37] in string theory frameworks. This case can

\footnotetext{
${ }^{11}$ It should be emphasized that we are calculating the entanglement entropy of the boundary state on the holographic screen, not the entropy associated with any bulk quantum fields. We refer to the degrees of freedom on the screen that govern the background gravitation dynamics as the gravitational degrees of freedom. Any low energy bulk excitations (which may include gravitons) are higher order corrections to the entanglement entropy and we do not discuss them.

${ }^{12}$ It is a logical possibility that a local theory could exhibit volume law entropy behavior due to open dynamics. Since the size of the leaf is constantly growing, there are degrees of freedom constantly being added to the system, which could already have long-range entanglement. This seems to be an ad hoc solution, and we do not elaborate on this possibility further.
} 
also be realized as a spin chain with interactions that couple all sites within a distance smaller than the characteristic nonlocal length scale. Above the nonlocal length scale an area law term starts to pick up and eventually dominates. However, because of this eventual turn-on of an area law, the qualitative features of the entropy normalized by volume are different than those exhibited by FRW entropy curves. Namely, the concavity of the $Q_{A}$ plot beyond the nonlocal length scale is opposite to that observed in the FRW case. This is because beyond the nonlocal length scale the entropy approaches an area law; hence the second derivative of $Q_{A}$ is positive, unlike that observed in the FRW case. Raising the temperature only adds an overall constant asymptotic value to $Q_{A}$. Hence, the concavity of $Q_{A}$ forbids the holographic theory of FRW spacetimes from being a theory with a characteristic nonlocal length scale smaller than the system size.

This reasoning leaves us with nonlocal theories with characteristic interaction lengths comparable to the system size - what does this mean? It simply means that a site can be coupled to any other site. For simplicity we consider SYK-like theories but rather than being zero dimensional we split up the degrees of freedom to live on a lattice but keep the random couplings between them. At first thought, one may think that because of the random, all-to-all coupling the entanglement entropy for all subregions would always be maximal. However this is not the case. The entanglement entropy for small regions is indeed maximal, but then deviates at large length scales $[39,40]$. One can intuitively understand this by thinking about the $\mathrm{SYK}_{2}$ model and Bell pairs. The SYK couplings are random, and some sites have significantly higher coupling than average. In the ground state, these pairs have a high probability of being entangled, so if the subregion of interest contains only half of one of these special pairs, this raises the entanglement with the outside. However, once the subregion becomes larger there is a higher probability that a complete Bell pair is contained, and this drops the entanglement entropy.

From this intuition, one can see that the ground state of SYK-like theories has near maximal entanglement for small regions, which then deviates at large length scales. At higher energies, the probability of minimizing the term in the Hamiltonian coupling these special sites (and creating the effective Bell pair) is lowered, and hence the entanglement entropy of all subregions monotonically increases [40,44]. This behavior is reminiscent of that observed in FRW entanglement entropy if we relate the fluid parameter, $w$, to the energy of the nonlocal state: the case (II) listed above. The limit of $T \rightarrow \infty$ then corresponds to $w \rightarrow-1$.

The third possibility (III) is similar to the one just discussed, but with the difference that $w$ is dual not to temperature but to a fundamental parameter dictating the "connectivity" of the boundary theory. In the language of
$\mathrm{SYK}_{q}$, this would correspond to changing $q$, where $q$ is the number of coupled fermions in each interaction term of the Hamiltonian. As $q$ increases, the ground state entanglement monotonically increases and as $q \rightarrow \infty$ becomes maximal. This would be the limit corresponding to $w \rightarrow-1$. However, any possibility like this, which employs a change of a fundamental parameter of the Hamiltonian, requires us to manufacture the whole Hilbert space of the boundary theory by considering the collection of only the low energy states for each value of $q$. We would like one related class of spacetimes to be dual to one boundary theory, which is not the case in this option. We thus focus on option (II) as the best candidate, but we cannot logically exclude option (III).

It is interesting to observe the relationship between where the deviation from volume law entropy occurs and where the corresponding spacetime emerges. In the Schwarzschild-AdS case, $Q_{A}$ drops from 1 immediately at small subregions, and the spacetime that emerges is precisely that which is reconstructed from small subregions. Hence the directly reconstructable region appears at the boundary and grows inwards as the temperature of the state is lowered. The converse is true in the case of FRW spacetimes. As we move away from $w=-1$, the entanglement entropy drops from maximal at large subregions and the corresponding spacetime that emerges is constructed by intersecting large surfaces. This is because the HRRT surfaces of small subregions of leaves with $w$ near -1 all lie on the same codimension- 1 surface, the future causal boundary of the leaf, analogous to the small surfaces in Fig. 13 in Appendix C 3. The HRRT surfaces for large subregions deviate from this and hence allow for reconstructing a codimension-0 region starting with points deepest in the bulk.

The language of quantum error correction [17] and tensor networks $[11,25,26]$ allows for a nice interpretation of this phenomenon. The loss of entanglement in pure gravitational degrees of freedom affords nature the opportunity to redundantly encode local bulk degrees of freedom in the boundary. In AdS spacetime, short range entanglement is lost first, and hence there is "room" for the information of local bulk degrees of freedom to be stored. In the case of FRW, long range entanglement is lost first, and subsequently points in the bulk that require large subregions to reconstruct emerge first.

\section{HOLOGRAPHIC HILBERT SPACES}

The analysis of the previous sections brings us to a suitable position to discuss the structure of holographic Hilbert spaces. In this section, we propose how a single theory can host states with different spacetime duals while keeping geometric operators linear in the space of microstates for a fixed semiclassical geometry. We use intuition gathered from quantum thermodynamic arguments to guide us. Similar ideas have been discussed in Ref. [45]. Here we 
present a slightly generalized argument to emphasize its independence of dynamics, and explain its application to our framework.

Let us assume that the entanglement entropy of subregions of a boundary state dual to a semiclassical geometry is calculated via the HRRT prescription. Given a bulk spacetime, one can then find the corresponding entanglement entropies for all subregions of the boundary. Note that here we consider the "classical limit." Namely, all the subregions we consider contain $O(\mathcal{N})$ degrees of freedom, where

$$
\mathcal{N}=\frac{\mathcal{A}}{4 l_{\mathrm{P}}^{d-1}},
$$

with $\mathcal{A}$ being the volume of the holographic space. The collection of all boundary subregions and their corresponding entanglement entropies is referred to as the entanglement structure of the state, which we denote by $S(|\psi\rangle)$.

From here, it is natural to ask whether or not all states with the same entanglement structure are dual to the same bulk spacetime. This might indeed be the case, but it leads to some undesirable features. These primarily stem from the fact that given a particular entanglement structure, one can find a basis for the Hilbert space in which all basis states have the specified entanglement structure. For a Hilbert space with a local product structure, one can do this by applying local unitaries to a state-these retain the entanglement structure and yet generate orthogonal states. This would imply that by generically superposing $e^{O(\mathcal{N})}$ of these states, one could drastically alter the entanglement structure and create a state dual to a completely different spacetime. Hence, geometric quantities could not be represented by linear operators, even in an approximate sense. If this were the case, a strong form of state dependence would be necessary to make sense of dynamics in the gravitational degrees of freedom [6].

However, it is not required that every state in the holographic Hilbert space with the same entanglement structure is dual to the same spacetime. How can this consistently happen? Given an entanglement structure, $S(|\phi\rangle)$, we expect the existence of a subspace in which generic states (within this subspace) have this same entanglement structure up to $O\left(\mathcal{N}^{p}\right)$ corrections with $p<1$. The existence of a subspace with a unique entanglement structure is not surprising if the dimension of the subspace is $e^{O\left(\mathcal{N}^{p}\right)}$ with $(p<1)$, since we generally expect

$$
S\left(\sum_{i=1}^{e^{M}} c_{i}\left|\psi_{i}\right\rangle\right)=S(|\psi\rangle)+O(M),
$$

where $S\left(\left|\psi_{i}\right\rangle\right)=S(|\psi\rangle)$ for all $i$.

However, we argue further that there exist such subspaces with dimension $e^{O(\mathcal{N})}$, spanned by some basis states $\left|\psi_{i}\right\rangle\left(i=1, \ldots, e^{Q \mathcal{N}}\right)$, with

$$
S\left(\sum_{i=1}^{e^{\mathcal{N}}} c_{i}\left|\psi_{i}\right\rangle\right)=S(|\psi\rangle)+O\left(\mathcal{N}^{p} ; p<1\right)
$$

where $Q \leq 1$ does not scale with $\mathcal{N}$. The existence of these subspaces with entanglement structures invariant under superpositions is expected from canonical typicality (also referred to as the general canonical principle) $[46,47]$. This provides us with the powerful result that generic states in subspaces have the same reduced density matrix for small subsystems (up to small corrections). The proof of this statement is purely kinematical and hence applies generally. In fact, from canonical typicality the correction term in Eq. (24) is exponentially small, $O\left(e^{-Q \mathcal{N} / 2}\right)$.

Canonical typicality is a highly nontrivial statement because the size of the subspaces in question is large enough that one would naively think that superpositions would ruin the entanglement structure at $O(\mathcal{N}){ }^{13}$ Therefore, even if one considers an exponentially large superposition of microstates (so long as they are generic states from the same subspace), geometric operators can be effectively linear within this subspace. We propose that states dual to semiclassical geometries are precisely generic states within their respective subspaces.

An example of one of these subspaces is an energy band of a SYK theory. These harbor an exponentially large number of states, and yet from canonical typicality any superposition of generic states within this band has the same entanglement entropy. Another example is states that have energy scaling with the central charge, $c$, in AdS/CFT. These are dual to large black holes and there are also an exponentially large number of states within the energy band. Despite this, generic states within this energy band have the same entanglement entropy structure. Essentially, canonical typicality proves the existence of exponentially large subspaces that have entanglement structures preserved under superpositions of just as many states.

We need this strengthened statement because the entanglement entropy calculations for FRW suggest that the size of subspaces dual to identical spacetimes is exponentially large. This is because the quantity $Q$ in Eq. (24) is related with von Neumann entropies characterizing the whole state, e.g. $Q_{A}$ in Sec. III A with $A$ being the half boundary space and $Q_{w}(\pi / 2)$ in Sec. III B. This intuition stems from the statement that the thermal entropy density and entanglement entropy density for states in the thermodynamic limit are approximately equal. For generic states within some energy interval subspace, this holds by canonical typicality. The statement also results from assuming that the system satisfies the ETH (like in AdS/CFT). SYK models, however, do not strictly satisfy the ETH; nevertheless, it

\footnotetext{
${ }^{13}$ Note that if one fine-tunes coefficients and selects states in this subspace carefully, one could construct a state with lower entanglement via superposition.
} 
remains true that $Q_{A}$ at half system size gives a good approximation for the thermal entropy density, and the discrepancy vanishes as the energy of the states is increased. For these reasons, we expect $Q_{w}(\pi / 2)$ to well approximate the thermal entropy density of states dual to a FRW spacetime with fluid parameter $w$.

We can now address the properties of typical states within an entire Hilbert space. Consider a holographic Hilbert space of a given theory, e.g. a CFT with a finite cutoff or the holographic theory of FRW spacetimes. If there are multiple superselection sectors in a given theory, then we focus on one of them. In such a Hilbert space, the effective subspace with $Q=1$ corresponds to typical states. Applying Page's analysis, we can then conclude that the only entanglement structure consistent with Eq. (24) where $Q=1$ must be that of maximal entropy. For example, the number of microstates for a large black hole approaches the dimension of the boundary Hilbert space as $T \rightarrow \Lambda$, and these states are maximally entangled. Similarly, using the argument in the previous paragraph, the number of independent microstates in the de Sitter limit approaches the dimension of the boundary Hilbert space, and these states are maximally entangled. As shown in Sec. II, the directly reconstructable spacetime region vanishes in these cases-an effective subspace with $Q=$ 1 does not have reconstructable spacetime. It is in this sense that typical states in the whole Hilbert space have no reconstructable spacetime.

On the other hand, if $Q<1$, the corresponding entanglement structure $S(|\psi\rangle)$ can be nonmaximal, and generic states in this subspace may be dual to some bulk spacetime. As discussed in Sec. III C, we expect that dynamics of the boundary theory can naturally select these subspaces, for example by simply lowering the energy of the system in the case of the boundary CFT.

The structure discussed here allows for a single holographic Hilbert space to harbor effective subspaces dual to different geometries, allows for a "generically linear" spacetime operator, and hence eliminates the need for any strong form of state dependence. ${ }^{14}$ Because this "spacetime operator" is identical for states of a given entanglement, it obviously acts linearly on generic superpositions of states within one of these dynamically selected,

\footnotetext{
${ }^{14} \mathrm{By}$ strong state dependence, we mean a theory that would require state dependence to describe bulk excitations in the directly reconstructable region of a boundary state which is a generic superposition of states dual to a given spacetime. For a more detailed analysis of this statement, we refer the reader to Ref. [6]. The main result is that requiring linearity for the multiple boundary representations of a bulk operator is impossible if the number of geometry microstates is $e^{\mathcal{N}}$. This prohibits the existence of a directly reconstructable region for typical states. Note that the directly reconstructable region does not probe behind black hole horizons, and hence we are not addressing the possibility that state dependence is necessary to recover the black hole interior.
}

entanglement-invariant subspaces. We suspect that it is only in this thermodynamic sense that classical spacetime emerges from the fundamental theory of quantum gravity.

\section{CONCLUSION}

\section{A. Discussion}

Our understanding of the relationship between spacetime and entanglement seems to be converging. The necessity of entanglement between boundary degrees of freedom for the existence of spacetime has been known for some time, but this fact may have mistakenly established the intuition that the fabric of spacetime itself is purely this entanglement. However, this cannot be the case. A one-to-one mapping between the entanglement structure of a boundary state and the directly reconstructable bulk spacetime cannot be upheld in a state independent manner. In addition, we see that as boundary entanglement approaches maximality the reconstructable region of the bulk vanishes.

In hindsight, this should not be too surprising. Let us recall Van Raamsdonk's discussion [12] relating spacetime to entanglement by examining the link between mutual information and correlations in a system. The mutual information between two boundary subsystems $A$ and $B$ is defined as

$$
I(A, B)=S(A)+S(B)-S(A \cup B) .
$$

This quantity bounds the correlations in a system between operators $\mathcal{O}_{A}$ and $\mathcal{O}_{B}$, supported solely on $A$ and $B$ via the relation

$$
I(A, B) \geq \frac{\left(\left\langle\mathcal{O}_{A} \mathcal{O}_{B}\right\rangle-\left\langle\mathcal{O}_{A}\right\rangle\left\langle\mathcal{O}_{B}\right\rangle\right)^{2}}{2\left|\mathcal{O}_{A}\right|^{2}\left|\mathcal{O}_{B}\right|^{2}}
$$

Hence, when the mutual information between two subregions $A$ and $B$ vanishes, the correlation between local operators supported within the subregions must also vanish. Assuming that subregion duality holds, this implies that correlation functions of bulk fields vanish. Generally, correlators between two bulk fields go as

$$
\left\langle\mathcal{O}_{1}\left(x_{1}\right) \mathcal{O}_{2}\left(x_{2}\right)\right\rangle \sim a f(L),
$$

where $L$ is the distance of the shortest geodesic connecting $x_{1}$ and $x_{2}, a$ is some theory dependent constant, and $f(z)$ is a decreasing function of $z$. One can then make the argument that decreasing entanglement between regions drops the mutual information between the regions, and hence makes $L$ effectively infinite. This implies that the spacetime regions dual to subregions $A$ and $B$ are disconnected when the entanglement (and hence mutual information) vanishes. For intuition's sake, one can imagine two subregions of the AdS boundary which are in a connected entanglement phaseincreasing the distance between these two subregions drops 
the mutual information. This is an argument demonstrating the need for entanglement in a holographic theory dual to spacetime, so long as the holographic theory has subregion duality.

However, there is a different (quite the opposite) way to make the mutual information between small (less than half of the system) subregions vanish, and consequently kill the bulk correlations. This is by considering maximally entropic boundary states - in these, the mutual information vanishes for any pair of subregions. This is the case both in cutoff temperature AdS black holes and in the de Sitter limit of the holographic theory of FRW universes. In these, the boundary states are maximally entropic and hence the bulk correlators must vanish; however, there exist finite length geodesics in the bulk (even if restricted only to the directly reconstructable region) which connect all points on the boundary. This means that the prefactor, $a$, of Eq. (27) must vanish, making the bulk theory ultralocal. In these cases, the maximal entropy implies that there cannot be an extra emergent bulk dimension. This is because the ground state of any quantum field theory quantized on spacelike hypersurfaces must be entangled at arbitrarily short scales, which is violated by the assumption that $a=0$. However, this is not necessarily unexpected-in both de Sitter space and cutoff temperature black holes, the directly reconstructable regions are codimension-1 null surfaces of the bulk (the de Sitter horizon and black hole horizon respectively). A natural description of the fields on these surfaces would be through null quantization, which is known to be ultralocal [48]. Accordingly, we see a breakdown in the holographic description.

From the above arguments one can convince oneself that it is not entanglement itself which allows for the construction of spacetime, but rather something related to intermediate entanglement.

How can this be better understood? The framework of tensor networks provides some intuition behind this. Here, a maximally entropic boundary state is most naturally represented by a single bulk node with one bulk leg and multiple boundary legs. ${ }^{15}$ Hence the spacetime is just one nonlocalizable bulk region, a "clump" as defined in Ref. [8]. This bulk point can be reconstructed once a subregion of the boundary contains more than half of the boundary legs. Here it is clear that a maximally entropic boundary state has no dual spacetime, and yet it is possible to encode a bulk code subspace with full recovery once more than half of the boundary is obtained. Note that these typical states all satisfy (in fact saturate) the holographic entropy cone inequalities [49] simply because a random tensor network accurately describes the state, but this does

\footnotetext{
${ }^{15}$ Any attempt to create a bulk by artificially including more nodes with extremely large bulk bond dimension can be reduced to the case of one bulk node.
}

not mean that there is a reconstructable region of the spacetime.

Additionally, if maximally entropic states did have reconstructable spacetime, then state dependence would be necessary in order to describe bulk excitations in these states, under the assumption that subregion duality holds. This is because the number of microstates with maximal entropy is approximately the dimension of the full boundary Hilbert space, and by the argument in Sec. VC of Ref. [6], it is impossible to find a boundary representation of a bulk operator that has support only on a subregion of the boundary and acts approximately linearly on all microstates of a given spacetime. Intuitively, this is because the operator will be overconstrained by insisting that it both has support on a subregion of the boundary and acts linearly on $D$ microstates, when the dimension of the full boundary space is $D$. This means that if we require state independence, then the only possible boundary operators representing bulk excitations for a maximally entropic state must have support on the full boundary space. ${ }^{16}$ Therefore, the minimum possible subregion in which bulk excitations can be encoded state independently is the whole boundary space; hence there is no directly reconstructable spacetime. This directly highlights the tension between reconstructing spacetime for maximally entropic states (in any manner), and requiring both subregion duality and state independence.

But what happens if we lower the entanglement of the boundary state while keeping the dimension of the boundary Hilbert space constant? Again, we turn to tensor networks for intuition. In these situations, a natural way to encode submaximal entanglement (while fixing the bulk leg dimension) is by including more bulk nodes. Therefore, by reducing the boundary entanglement, it is possible to create a bulk code subspace in which subsystem recovery is possible. It seems that quantum gravity naturally utilizes this submaximal entanglement in order to encode information via subregion duality. This suggests that perhaps entanglement is not the fundamental constituent of spacetime per se, but rather the avenue by which subregion duality manifests.

\section{B. Future directions}

This paper has attempted to clarify the nature of spacetime in holographic theories and it naturally raises interesting questions to be investigated in future work.

\footnotetext{
${ }^{16}$ This is not contradicting the statement in the previous paragraph that the sole bulk node's state in a random tensor can be recovered with just more than half of the boundary. In that case, only the recovery of the bulk code subspace for one microstate was considered. State independence would require us to have an operator that acts linearly on all microstates of a given spacetime.
} 


\section{Reconstructability and generalized holographic renormalization}

The analysis of this paper utilized the condition for reconstructable spacetime presented in Ref. [8], but appropriately generalized for use in the context of holographic screens [6]. This paper illuminated some highly desirable properties of the directly reconstructable region defined in this manner-namely that one can describe this region state independently. It would be extremely beneficial to attempt to find an explicit way to construct bulk operators using this method, perhaps uniting it with the methods of entanglement wedge reconstruction [50,51].

It would also be interesting to try and develop new tools for reconstructing the bulk. The relationship between the depth in the bulk and the scale in the boundary theory in AdS/CFT suggests that it may be possible to define the reconstructable region of spacetime as that which is swept through a renormalization procedure. How this manifests in general holography is not clear, but it is suggestive that there exists at least one foliation where one can "pull" the leaf inward while retaining the ability to consistently apply the HRRT prescription. Because the area of these renormalized leaves is monotonically decreasing, it is natural that this "pulling" may correspond to some renormalization procedure. The decrease in area also happens locally, which can be seen by generalizing the spacelike monotonicity theorem of Ref. [5].

One guess as to how to construct the renormalized leaf is to first pick the coarse graining scale of the boundary, and then define the new leaf as the collection of all of the deepest points of the extremal surfaces anchored to subregions with the size of the coarse graining scale. In AdS/ CFT this will pull the boundary in along the $z$ direction as expected, while in FRW spacetimes this will pull the leaf along the null direction if the coarse graining scale is small. Using this method, one can renormalize to a given scale in a number of different ways. For example, one could perform many small renormalization steps or one large one. The renormalized leaves in the two cases will generically differ, and this may correspond to the difference between one-shot renormalization and a renormalization group method. The collection of all renormalized leaves may then determine the reconstructable region. ${ }^{17}$ Theorem 1 tells us that once the renormalized state becomes maximally entropic, the renormalization procedure must halt. Furthermore, because extremal surfaces for nonmaximally entropic states probe the bulk, this renormalization procedure continues until the leaf becomes maximally entangled. Thus, this renormalization group flow halts only once a bifurcation surface or a null nonexpanding surface is reached. In this language,

\footnotetext{
${ }^{17}$ Using this construction, it is not possible to extend reconstruction beyond horizons, but it is possible to reach behind entanglement shadows.
}

maximally entropic states correspond to fixed points. This is speculation, but may shed some light on the nature of renormalization in general holographic theories.

\section{Cosmic equilibration}

In Sec. II E, we proved that maximally entropic states have no directly reconstructable spacetime. Additionally, we argued that if one desires a state on a holographic screen to be maximally entropic and evolve in time, then the holographic screen is a null nonexpanding surface and the directly reconstructable region is no more than the screen itself. This suggests that in a holographic theory of cosmological spacetimes, if a state becomes maximally entropic and the screen does not halt, then the holographic description approaches that of de Sitter space. Consequently, the area of the screen is constant. It would be interesting to investigate the result from the other direction. By first assuming that the screen approaches a constant area, one may be able to argue that the leaves would then approach maximal entropy, and hence the holographic description approaches that of de Sitter space. This could provide another way to consider equilibrating to de Sitter-type solutions; see Ref. [52].

\section{Complementarity}

In Appendix A, we highlighted the dependence of the reconstructable region on the frame of reference. In the case of the two-sided AdS black hole, we considered different reference frames corresponding to different time slicings in the same boundary theory-as one shifts the difference in the two boundary times, one recovers more and more of the black hole interior. This is an example of complementarity. It would be interesting to pursue this idea further and investigate the directly reconstructable region of a twosided black hole.

One intriguing aspect of the two-sided black hole is that the directly reconstructable region does not extend beyond the extremal surface barrier; this is a macroscopic distance away from the future singularity, regardless of the boundary frame. Does this mean that the boundary CFT cannot describe semiclassical physics behind this barrier, even where curvature is small? Perhaps this means that there is a different description for the interior, living on a different holographic space.

\section{Fundamentality of subregion duality}

In many of the discussions throughout this paper, we either required subregion duality or saw that it naturally arose from other considerations. This seems to suggest that subregion duality is a fundamental characteristic of general holography. Investigating the manner in which subregion duality arises in AdS/CFT may shed light on holography in general spacetimes. 


\section{Holographic theory of flat FRW spacetimes}

One of the most obvious open problems is that of finding an effective holographic theory applicable beyond asymptotically AdS spacetimes. In this paper and throughout previous work, we have focused on the case of flat FRW universes and assumed that a theory exists on the holographic screen in which the generalized HRRT prescription holds. Investigations into this have led to a deeper understanding of the nature of entanglement in constructing spacetime, along with (the lack of) state dependence in holographic theories.

It seems that a consistent theory is possible, and the most promising candidate for a theory describing the gravitational degrees of freedom is a theory with longrange interactions in which the energy of the states is dual to the fluid parameter of the FRW universe. We know that it cannot be entirely nonlocal because this would prohibit the existence of entanglement phase transitions. A theory with long-range interactions would accurately reproduce the entanglement entropy structure we observe for FRW universes and would allow for a universal theory describing the single class of spacetimes. Beyond this, we have some additional data about the properties of the boundary theory.

We know that a code subspace of states manifests, and these states are dual to bulk excitations. Assuming subregion duality holds, one can ask the question of whether or not nonlocality/very long-range interaction in the gravitational degrees of freedom prohibits the local propagation of bulk excitations in the boundary theory. We expect that the operators dual to bulk excitations are weakly coupled to the gravitational degrees of freedom, and that a local description of these bulk operators exists in the boundary. In fact, this is what happens when one renormalizes the AdS boundary down to a single AdS volume [53]. This renormalization induces an infinite set of interactions which makes the resulting theory on the renormalized boundary nonlocal. Despite this, the renormalized theory still describes bulk physics through subregion duality. Hence, the nonlocality of the boundary theory does not seem to be a fundamental obstacle in describing low energy excitations using local dynamics in the boundary theory. ${ }^{18}$ The dynamics of boundary operators dual to bulk excitations in flat FRW spacetimes was studied in Ref. [54] and it was determined that regardless of dimension and fluid parameter, the spread of these operators was characteristic of a theory with $z=4$ Lifshitz scaling. This provides extra constraints for finding a candidate theory.

\footnotetext{
${ }^{18}$ It would be interesting to study this effective boundary theory, induced in AdS/CFT by renormalizing all the way down to the AdS scale. The holographic theory capturing sub-AdS locality could be very closely related to the theory on holographic screens.
}

\section{Holographic theory for general spacetimes}

It might appear that defining quantum gravity using holography, as envisioned here, is background dependent. Namely, the holographic theory is given for each class of background spacetimes, e.g. asymptotically AdS spacetimes and flat FRW spacetimes. This situation is analogous to defining string theory on the world sheet, which is defined separately on each target space background. From the perspective of the world sheet, different backgrounds correspond to different theories living on the two-dimensional spacetime. Nevertheless, we believe there exists some unified framework encompassing all these possibilities. Similarly, in the case of holographic theories, it is plausible that the resultant theories for different background spacetimes correspond to different sectors described within a single framework.

\section{ACKNOWLEDGMENTS}

We thank Chris Akers, Francisco Machado, Sanjay Moudgalya, and Fabio Sanches for useful discussions. This work was supported in part by the National Science Foundation under Grant No. PHY-1521446, by the Department of Energy, Office of Science, Office of High Energy Physics under Contract No. DE-AC02-05CH11231, and by MEXT KAKENHI Grant No. 15H05895.

\section{APPENDIX A: RECONSTRUCTABILITY OF TWO-SIDED BLACK HOLES AND COMPLEMENTARITY}

In the main part of the text, we have focused on spacetimes having a simply connected boundary. It is interesting to consider when this is not the case and examine which (if any) results persist. For definiteness, we here analyze the case of a two-sided eternal black hole in asymptotically AdS spacetime. In this case, the holographic screen is the union of the two asymptotic boundaries at spacelike infinity. The boundary theory comprises two CFTs, $\mathrm{CFT}_{L}$ and $\mathrm{CFT}_{R}$, which are decoupled from each other. Hence, the Hamiltonian for the system is given by

$$
H_{\text {total }}=H_{L}+H_{R}
$$

The times $t_{L}$ and $t_{R}$ associated respectively with $H_{L}$ and $H_{R}$ run in opposite directions along the two asymptotic boundaries.

Since the theories are decoupled, it might appear that one could evolve each of the theories independentlyeffectively foliating the holographic screen by two independent parameters, $\left(t_{L}, t_{R}\right)$. Per the construction outlined in Sec. II A, the directly reconstructable region would then be the union of all points localized by intersecting entanglement wedges of HRRT surfaces individually anchored to "one" leaf, each of which is labeled by $\left(t_{L}, t_{R}\right)$. Here, one 
leaf corresponds to picking a connected, equal time slice of the left boundary and independently a connected, equal time slice of the right boundary. If this were the case, the reconstructable region would be most of the spacetime, including a macroscopic portion of the interior (aside from a region near the singularity with $r<r_{+} / 2^{1 / d}$, where $r_{+}$is the horizon radius) [55].

However, a theory described by Hamiltonian dynamics should have a single time parameter. To make the holographic theory compatible with this, we postulate that there is a single parameter $t$ that foliates the multiple disconnected components of the holographic screen. From this assumption, there are multiple suitable foliations, and among them we must pick one-this corresponds to choosing a reference frame, a gauge for the holographic redundancy [56]. In the case of a two-sided black hole, this gives us a one parameter family of foliations corresponding to the freedom in choosing the relative time shift between $t_{L}$ and $t_{R}$ in the CFTs, even after choosing a natural foliation at each boundary.

In general, each of these individual foliations reconstructs a different region of the bulk spacetime. For example, adopting the usual thermofield double state construction [57] corresponds to choosing a reference frame

$$
t_{L}=t_{R}=t,
$$

in which the $t=0$ slice in the bulk is the one passing through the bifurcation surface. Since time translation is a Killing symmetry in this spacetime, and the bifurcation surface is invariant under this translation, the HRRT surfaces for any time $t$ never enter the interior of the black hole. Connected HRRT surfaces always pass through the bifurcation surface in such a situation (unless the subregion has support on only one of the boundaries, in which case the HRRT surface stays in one side of the black hole). The reconstructable region in this reference frame, therefore, does not include the interior of the black hole.

However, one could alternatively consider a reference frame in which there is a relative shift in the two times

$$
t_{L}=t+\Delta, \quad t_{R}=t .
$$

In this case, the connected HRRT surfaces would not necessarily pass through the bifurcation surface and could probe regions of the interior, and hence parts of the interior will be reconstructable. We can interpret this foliation dependence of the reconstructable region as a version of complementarity [58]. In this light, the canonical thermofield double time foliation corresponds to an entirely exterior description of the black hole, while increasing $\Delta$ allows for more of the region behind the horizon to be reconstructed. An important point is that we should not consider leaves with different $\Delta$ 's in a single description-they correspond to different descriptions in different reference frames. We also note that regardless of the foliation, we cannot reconstruct near the singularity because of the extremal surface barrier located at $r=r_{+} / 2^{1 / d}$. This suggests that in order to probe physics of the singularity we must use a different method.

With this interpretation of bulk reconstruction, we examine whether or not spacetime disappears as we approach maximal entropy. A priori, it seems that a macroscopic spacetime region would remain as we increase the black hole radius because some portion of the interior is reconstructable. However, this apparent contradiction is resolved by considering a finite coordinate time interval and examining the reconstructable volume as one increases the temperature.

Consider any foliation where the relative time shift between $t_{L}$ and $t_{R}$ has been fixed. In order to carry out the analysis analogous to Sec. II B, we fix an interval of coordinate time $\Delta t$ and fix the cutoff surface at $r=R$. Increasing the temperature of the black hole moves the horizon closer and closer to the cutoff surface, which can be represented in the Penrose diagram as in Fig. 10. The allowed range of times is depicted by the constant time surfaces $t_{1}$ and $t_{2}$. As we take the limit $r_{+} \rightarrow R$, which corresponds to taking the temperature of the black hole $T_{\mathrm{H}} \rightarrow \Lambda$ where $\Lambda$ is the cutoff in the boundary theory, the finite range of time collapses down to the bifurcation surface on both sides. Thus, the relative reconstructable spacetime volume shrinks to 0 .

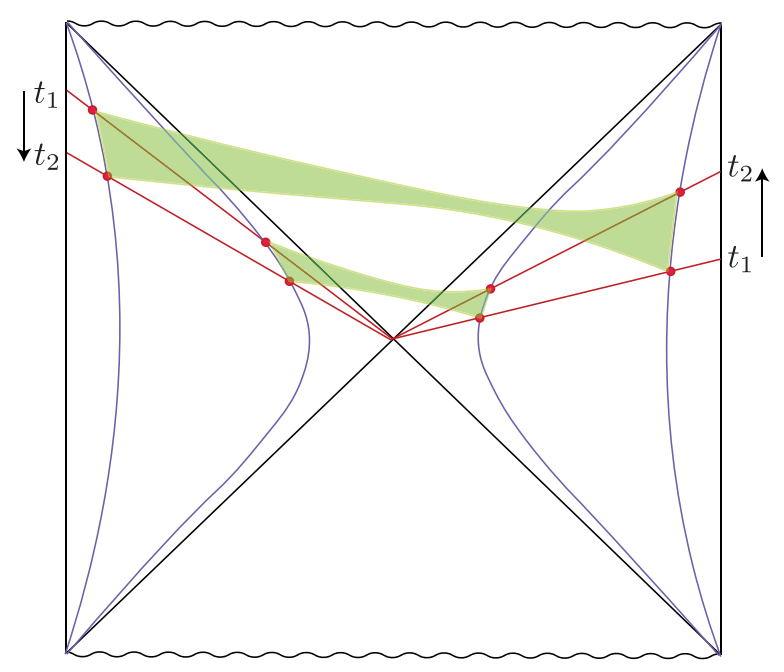

FIG. 10. The spacetime regions reconstructable using connected HRRT surfaces anchored to subregions with support on both asymptotic boundaries within the range $t \in\left[t_{1}, t_{2}\right]$ are depicted (green shaded regions) for two different values of black hole horizon radius $r_{+}$in a two-sided eternal AdS black hole. The holographic screen (blue) in both cases is the cutoff surface $r=R$. Here, we superimpose the respective Penrose diagrams in the two cases to compare the amount of reconstructable spacetime volume available by allowing connected HRRT surfaces. 
We find that our claim persists despite the addition of a disconnected boundary region that allows for the reconstruction of spacetime behind a black hole horizon.

\section{APPENDIX B: CALCULATIONS FOR THE SCHWARZSCHILD-ADS SPACETIME}

In this appendix, we provide explicit calculations of the spatial volume and HRRT surfaces of the SchwarzschildAdS spacetime.

\section{Reconstructable volume}

The Schwarzschild-AdS spacetime in $d+1$ dimensions is described by the metric

$d s^{2}=-\left(\frac{r^{2}}{l^{2}}+1-\frac{2 \mu}{r^{d-2}}\right) d t^{2}+\frac{d r^{2}}{\frac{r^{2}}{l^{2}}+1-\frac{2 \mu}{r^{d-2}}}+r^{2} d \Omega_{d-1}^{2}$,

where $l$ is the AdS radius, and $\mu$ is related with the black hole horizon radius $r_{+}$as

$$
2 \mu=\frac{r_{+}^{d}}{l^{2}}\left(1+\frac{l^{2}}{r_{+}^{2}}\right) .
$$

The Hawking temperature of the black hole is given by

$$
T_{\mathrm{H}}=\frac{d r_{+}^{2}+(d-2) l^{2}}{4 \pi r_{+} l^{2}} .
$$

Consider a large AdS black hole $r_{+} \gg l$. In this limit,

$$
2 \mu=\frac{r_{+}^{d}}{l^{2}}, \quad T_{\mathrm{H}}=\frac{d r_{+}}{4 \pi l^{2}},
$$

and the metric is well approximated by

$$
d s^{2}=-\left(\frac{r^{2}}{l^{2}}-\frac{r_{+}^{d}}{l^{2} r^{d-2}}\right) d t^{2}+\frac{d r^{2}}{\frac{r^{2}}{l^{2}}-\frac{r^{d}}{l^{2} r^{d-2}}}+r^{2} d \Omega_{d-1}^{2} .
$$

Let us now introduce an infrared cutoff $r \leq R$ and consider the spatial volume between the black hole horizon and the cutoff

$$
\begin{aligned}
V\left(r_{+}, R\right) & =A_{d-1} \int_{r_{+}}^{R} \frac{r^{d-1}}{\sqrt{\frac{r^{2}}{l^{2}}-\frac{r_{+}^{d}}{l^{2} r^{d-2}}}} d r \\
& =\frac{2 \pi^{d / 2}}{\Gamma(d / 2)} l_{+}^{d-1} \int_{1}^{\frac{R}{r_{+}}} \frac{x^{d-2}}{\sqrt{1-\frac{1}{x^{d}}}} d x,
\end{aligned}
$$

where $A_{d-1}=2 \pi^{d / 2} / \Gamma(d / 2)$ is the area of the $(d-1)$ dimensional unit sphere. Here, we have focused on the spatial volume because the system is static.
We normalize this volume by the volume of the region $r \leq R$ in empty AdS spacetime,

$$
\begin{aligned}
V(R) & =A_{d-1} \int_{0}^{R} \frac{r^{d-1}}{\sqrt{\frac{r^{2}}{l^{2}}}+1} d r \\
& =\frac{2 \pi^{d / 2}}{(d-1) \Gamma(d / 2)} l R^{d-1},
\end{aligned}
$$

where we have used $R \gg l$ in the second line. This gives us the quantity quoted in Eq. (1),

$$
f\left(\frac{r_{+}}{R}\right) \equiv \frac{V\left(r_{+}, R\right)}{V(R)}=(d-1) \frac{r_{+}^{d-1}}{R^{d-1}} \int_{1}^{\frac{R}{r_{+}}} \frac{x^{d-2}}{\sqrt{1-\frac{1}{x^{d}}}} d x .
$$

\section{HRRT surfaces}

Consider a large black hole in asymptotically AdS spacetime. The holographic theory is then a CFT. Suppose the temperature of the system $T$ is lower than the cutoff scale, $T<\Lambda$. Here we study the behavior of the von Neumann entropy of a spherical cap region $A$ on $r=R$ in this setup.

The region is specified by a half opening angle $\psi$,

$$
0 \leq \theta \leq \psi,
$$

where $\theta$ is a polar angle parametrizing $S^{d-1}$ with constant $t$ and $r$. The HRRT surface $\gamma_{A}$ is then given by function $r(\theta)$, which is determined by minimizing the area functional,

$$
\left\|\gamma_{A}\right\|=\min _{r(\theta)}\left[A_{d-2} \int_{0}^{\psi} r^{d-2} \sin ^{d-2} \theta \sqrt{r^{2}+\frac{\left(\frac{d r}{d \theta}\right)^{2}}{\frac{r^{2}}{l^{2}}+1-\frac{2 \mu}{r^{d-2}}}} d \theta\right],
$$

with the boundary condition

$$
r(\psi)=R,
$$

where $A_{d-2}$ is the area of the $(d-2)$-dimensional unit sphere, and $\mu$ is given by Eq. (B1). Here and below, we assume $\psi \leq \pi / 2$. For $\psi>\pi / 2$, the entropy of $A$ is determined by $S(\psi)=S(\pi-\psi)$.

The surface $\gamma_{A}$ is well approximated to consist of two components: (i) a "cylindrical" piece with $\theta=\psi$, which is perpendicular to the cutoff surface $r=R$ and extends down to $r=r_{0}(<R)$, and (ii) the "bottom lid" with $r=r_{0}$ and $0 \leq \theta \leq \psi$; see Fig. 11. The area of the surface is then given by 


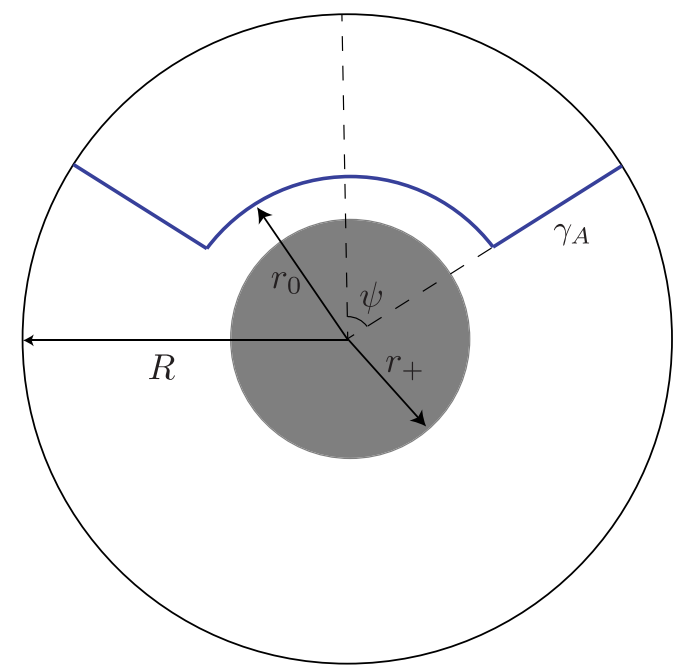

FIG. 11. The HRRT surface $\gamma_{A}$ in the Schwarzschild-AdS spacetime can be well approximated by consisting of two components: a cylindrical piece with $\theta=\psi$ and a bottom lid piece with $r=r_{0}$.

$$
\begin{aligned}
\left\|\gamma_{A}\right\|= & \min _{r_{0}}\left[A_{d-2} \sin ^{d-2} \psi \int_{r_{0}}^{R} \frac{r^{d-2}}{\sqrt{\frac{r^{2}}{l^{2}}-\frac{r_{+}^{d}}{l^{2} r^{d^{-2}}}}} d r\right. \\
& \left.+A_{d-2} r_{0}^{d-1} \int_{0}^{\psi} \sin ^{d-2} \theta d \theta\right],
\end{aligned}
$$

where $r_{+}$is the horizon radius, and we have used the approximation that $r_{+} \gg l$ and hence Eq. (B4). The value of $r_{0}$ is determined by the minimization condition

$$
\sqrt{r_{0}^{2}-\frac{r_{+}^{d}}{r_{0}^{d-2}}}=\frac{\sin ^{d-2} \psi}{(d-1) \int_{0}^{\psi} \sin ^{d-2} \theta d \theta} l .
$$

As discussed in Sec. II B, the cutoff at $r=R$ in our context simply means that the renormalization scale in the boundary theory is lowered; in particular, it does not mean that the theory is modified by actually terminating space there. The length in the boundary theory, therefore, is still measured in terms of the $d$-dimensional metric at infinity, $r=\infty$, with the conformal factor stripped off. The radius of the region $A$ is then given by

$$
L=l \psi,
$$

and not $R \psi$. Since the cutoff length is $1 / \Lambda \approx l^{2} / R$, we should only consider the region $\psi \gtrsim l / R$.

The solution of Eq. (B13) behaves as

$$
\begin{array}{r}
\text { (i) } r_{0}=\frac{l}{\psi}\left(\gg r_{+}\right) \text {for } \frac{l}{R}<\psi \ll \frac{l}{r_{+}}, \\
\text {(ii) } r_{0}-r_{+}=\frac{l^{2}}{d \psi^{2} r_{+}}\left(\ll \frac{r_{+}}{d}\right) \text { for } \frac{l}{r_{+}} \ll \psi \ll 1,
\end{array}
$$

$$
\text { (iii) } r_{0}-r_{+}=O(1) \frac{l^{2}}{r_{+}} \text {for } \psi \approx O(1)
$$

In the case of (i), $\left\|\gamma_{A}\right\|$ is dominated by the first term in Eq. (B12), so that

$$
\left\|\gamma_{A}\right\|=\frac{A_{d-2}}{d-2} l R^{d-2} \psi^{d-2}
$$

Here and below, we assume $d>2$. We thus obtain an area law for the entropy,

$$
S_{A}=\frac{\left\|\gamma_{A}\right\|}{4 l_{\mathrm{P}}^{d-1}} \approx c A_{d-2} L^{d-2} \Lambda^{d-2},
$$

where $c \approx\left(l / l_{\mathrm{P}}\right)^{d-1}$ is the central charge of the boundary CFT.

In the case of (ii), $\left\|\gamma_{A}\right\|$ is given by

$$
\left\|\gamma_{A}\right\|=\frac{A_{d-2}}{d-2} l R^{d-2} \psi^{d-2}+\frac{A_{d-2}}{d-1} r_{+}^{d-1} \psi^{d-1} .
$$

We find that the first (second) term is larger for

$$
\psi<(>) \frac{d-1}{d-2} \frac{l R^{d-2}}{r_{+}^{d-1}},
$$

so that the entanglement entropy behaves as

$S_{A} \approx \begin{cases}c A_{d-2} L^{d-2} \Lambda^{d-2} & \text { for } L \ll L_{*}, \\ c A_{d-2} \frac{r_{+}^{d-1} L^{d-1}}{l^{2-2}} \approx c\left(\frac{T}{\Lambda}\right)^{d-1} A_{d-2} L^{d-1} \Lambda^{d-1} & \text { for } L \gg L_{*},\end{cases}$

where

$$
L_{*} \approx \frac{l^{2} R^{d-2}}{r_{+}^{d-1}} \approx \frac{\Lambda^{d-2}}{T^{d-1}}
$$

For $\psi \approx O(1)$, i.e. case (iii), we find

$$
S_{A} \approx c\left(\frac{T}{\Lambda}\right)^{d-1} A_{d-2} L^{d-1} \Lambda^{d-1} .
$$

Combining the results in all three cases gives the expression in Eqs. (17) and (18).

\section{APPENDIX C: CALCULATIONS FOR THE DE SITTER LIMIT OF FRW UNIVERSES}

This appendix collects explicit calculations for entropies and HRRT surfaces in the de Sitter limit of FRW spacetimes. 


\section{Entropies in the case of $(2+1)$-dimensional bulk}

Here we see that for $(2+1)$-dimensional FRW spacetimes, the results of Ref. [5] immediately tell us that the entanglement entropy of an arbitrary (not necessarily connected) subregion $A$ is maximal in the de Sitter limit,

$$
S_{A, w \rightarrow-1}=\frac{1}{4 l_{\mathrm{P}}} \min \{\|A\|,\|\bar{A}\|\} .
$$

Consider a FRW universe in $d+1$ dimensions dominated by a single ideal fluid component with the equation of state parameter $w=p / \rho(|w| \leq 1)$. From the analysis of Ref. [5], we know that the holographic entanglement entropy of a spherical cap region $A$ on a leaf-parametrized by the half opening angle $\psi$ as viewed from the center of the bulk-scales with the smaller of the volumes of $A$ and $\bar{A}$. The proportionality constant,

$$
Q_{w}(\psi) \equiv \frac{S(\psi)}{\frac{1}{4 l_{\mathrm{P}}^{d-1}} \min \{\|A\|,\|\bar{A}\|\}},
$$

satisfies the properties

$$
\begin{array}{r}
Q_{w}(\psi \rightarrow 0) \rightarrow 1, \quad Q_{w \rightarrow-1}(\psi) \rightarrow 1, \quad(\mathrm{C} 3) \\
\left.\frac{\partial Q_{w}(\psi)}{\partial \psi}\right|_{\psi=0}=0,\left.\quad \frac{\partial Q_{w}(\psi)}{\partial \psi}\right|_{\psi<\frac{\pi}{2}} \leq 0, \quad \frac{\partial Q_{w}(\psi)}{\partial w}<0 .
\end{array}
$$

[The original analysis was performed for $(3+1)$ dimensional FRW universes, but these properties persist in arbitrary spacetime dimensions.]

The second relation in Eq. (C3) implies that in the de Sitter limit, $w \rightarrow-1$, the holographic entanglement entropy of a spherical cap region is maximal. Now, consider $(2+1)$-dimensional FRW universes, in which a leaf has only one spatial dimension. We consider a subregion on the leaf consisting of the union of two small intervals $A$ and $B$. Note that a similar setup is often discussed in AdS/CFT, where two possible extremal surfaces homologous to the subregion compete, so that a phase transition from the disconnected to connected HRRT surfaces occurs as the regions $A$ and $B$ are taken to be closer; see Fig. 12. We want to understand what happens in the case of FRW spacetimes.

We denote the areas of two possible extremal surfaces by

$$
\begin{aligned}
E_{\text {disconnected }}(A B) & =E(A)+E(B) \\
& =Q_{w}(A)\|A\|+Q_{w}(B)\|B\|,
\end{aligned}
$$

and

$$
\begin{aligned}
E_{\text {connected }}(A B) & =E(A B C)+E(C) \\
& =Q_{w}(A B C)\|A B C\|+Q_{w}(C)\|C\|,
\end{aligned}
$$

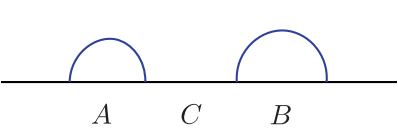

(a)

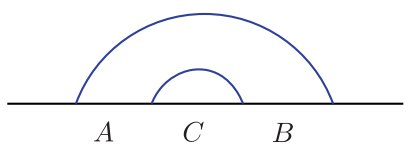

(b)
FIG. 12. Two possible extremal surfaces anchored to the boundary of a subregion $A B$ on a leaf, given by the union of two disjoint intervals $A$ and $B$. The areas of the surfaces depicted in (a) and (b) are denoted by $E_{\text {disconnected }}(A B)$ and $E_{\text {connected }}(A B)$, respectively.

where $A, B$, and $C$ are defined in Fig. 12. A phase transition can occur when

$$
E_{\text {disconnected }}(A B)=E_{\text {connected }}(A B) \text {. }
$$

The condition of Eq. (C7) can be satisfied for any $w$ away from the de Sitter limit because of the second relation in Eq. (C4). Since a larger region has a greater volume but also has a smaller coefficient, it is possible for the two extremal surfaces to compete. However, in the de Sitter limit the requirement for a phase transition becomes

$$
\|A B C\|+\|C\|=\|A\|+\|B\|,
$$

which is clearly impossible because the left-hand side is always greater. Since a general subregion of the leaf is a union of disconnected intervals, the above argument implies that the entanglement entropy is merely the sum of each interval's volume for sufficiently small regions. Extending the argument to large regions in which their complements matter, we can conclude that arbitrary subregions have maximal entanglement entropies in a $(2+1)$ dimensional de Sitter universe.

\section{Entropies in the $w \rightarrow-1$ limit of FRW spacetimes}

The global spacetime structure in the case of a single fluid component with $w \neq-1$ is qualitatively different from the case discussed mainly in Sec. II C, i.e. the case in which a universe approaches de Sitter space at late times. Nevertheless, here we show that the holographic entanglement entropy of an arbitrary subregion on a leaf becomes maximal in the $w \rightarrow-1$ limit.

Let us consider a FRW universe filled with a single fluid component with the equation of state $w$. The scale factor is then given by

$$
a(t)=c t^{\frac{2}{d(1+w)}},
$$

where $c>0$ is a constant. We focus on a leaf $\sigma_{*}$ at time $t_{*}$ and the causal region $D_{\sigma_{*}}$ associated with it. Following Ref. [5], we perform $t_{*}$-dependent coordinate transformation on the FRW time and radial coordinates $t$ and $r$, 


$$
\begin{gathered}
\eta=\frac{2}{d-2+d w}\left\{\left(\frac{t}{t_{*}}\right)^{\frac{d-2+d w}{d(1+w)}}-1\right\}, \\
\rho=\frac{2}{d(1+w)} c t_{*}^{-\frac{d-2+d w}{d(1+w)}} r .
\end{gathered}
$$

This converts the metric into the form

$$
\begin{aligned}
d s^{2}= & \left(\frac{\mathcal{A}_{*}}{A_{d-1}}\right)^{\frac{2}{d-1}}\left(\frac{d-2+d w}{2} \eta+1\right)^{\frac{4}{d-2+d w}} \\
& \times\left(-d \eta^{2}+d \rho^{2}+\rho^{2} d \Omega_{d-1}^{2}\right)
\end{aligned}
$$

where $A_{d-1}$ is the area of the $(d-1)$-dimensional unit sphere, defined below Eq. (B6), and $\mathcal{A}_{*}$ is the volume of the leaf $\sigma_{*}$

$$
\mathcal{A}_{*}=\left(\frac{d(1+w)}{2}\right)^{d-1} A_{d-1} t_{*}^{d-1}
$$

In these coordinates, $D_{\sigma_{*}}$ is mapped into the region $\eta \in$ $[-1,1]$ and $\rho \in[0,1-|\eta|] .{ }^{19}$

We can now take $w=-1+\epsilon$ in Eq. (C12) and expand it around $\epsilon=0$. This gives

$$
\begin{aligned}
d s^{2}= & \left(\frac{\mathcal{A}_{*}}{A_{d-1}}\right)^{\frac{2}{d-1}}\left(\frac{1}{(1-\eta)^{2}}\right. \\
& \left.-d \frac{\eta+(1-\eta) \ln (1-\eta)}{(1-\eta)^{3}} \epsilon+\cdots\right) \\
& \times\left(-d \eta^{2}+d \rho^{2}+\rho^{2} d \Omega_{d-1}^{2}\right) .
\end{aligned}
$$

The leading order term describes the causal region inside a leaf of volume $\mathcal{A}_{*}$ in de Sitter space with conformal coordinates. The time translational Killing symmetry in these coordinates is

$$
\begin{gathered}
\eta \rightarrow a \eta+1-a, \\
\rho \rightarrow a \rho .
\end{gathered}
$$

The expansion in Eq. (C14) is not valid when $\eta \lesssim 1-\epsilon$. However, this occurs only for a small subset of all the subregions on $\sigma_{*}$, which becomes measure 0 when $\epsilon \rightarrow 0$. Continuity then tells us that the entanglement entropy $S_{A}$ of any subregion $A$ on $\sigma_{*}$ takes the same value as that calculated in de Sitter space in the $\epsilon \rightarrow 0$ limit. However, we have already concluded from the argument in Sec. II C that the entanglement entropies take the maximal form in de Sitter space, hence

\footnotetext{
${ }^{19}$ For $w \geq-1+4 / d$, the region $D_{\sigma_{*}}$ hits the big bang singularity, so we need to restrict our attention to a portion of $D_{\sigma_{*}}$, e.g. $D_{\sigma_{*}}^{+}=\left\{p \in D_{\sigma_{*}} \mid t(p) \geq t_{*}\right\}$. This issue is not relevant to our discussion here.
}

$$
S_{A} \underset{w \rightarrow-1}{\longrightarrow} \frac{1}{4 l_{\mathrm{P}}^{d-1}} \min \{\|A\|,\|\bar{A}\|\}
$$

Note that the area of the leaf, $\mathcal{A}_{*}$, keeps growing indefinitely, so that $D_{\sigma_{*}}$ at each time $t_{*}$ is mapped to a different auxiliary de Sitter space. The ratio $Q_{w}(A)=$ $S_{A} /\left(\min \{\|A\|,\|\bar{A}\|\} / 4 l_{\mathrm{P}}^{d-1}\right)$, however, depends only on $w$ and not $t_{*}$.

\section{HRRT surfaces}

Here we present two examples in which one can analytically see the convergence of the HRRT surfaces onto the future boundary of the causal region of a leaf in the de Sitter limit.

\section{a. The de Sitter limit of FRW universes in $2+1$ dimensions}

As the first example, consider the de Sitter limit of FRW universes in $2+1$ dimensions,

$$
d s^{2}=a^{2}(\eta)\left(-d \eta^{2}+d x^{2}+d y^{2}\right) .
$$

Here, $\eta \in(-\infty, 0)$ is the conformal time, and the scale factor is given by

$$
a(\eta)=\frac{c}{\eta}
$$

where $c$ is a positive constant. In this case, we can obtain an analytic solution for HRRT surfaces, which are geodesics in $2+1$ dimensions.

In order to find a spacelike geodesic anchored to two points on the leaf, we can use the symmetry of the problem to rotate our axes so that the points lie at constant $y=y_{0}$. To find a geodesic, we need to extremize the distance functional

$$
\mathcal{D}=\int d \eta \frac{c}{\eta} \sqrt{\dot{x}^{2}-1}
$$

where $\dot{x}=d x / d \eta$, and we have used the fact that the geodesic lies on the $y=y_{0}$ hypersurface. This functional has no explicit dependence on $x$, which means the existence of a quantity that is conserved along the geodesic

$$
\frac{\partial \mathcal{D}}{\partial \dot{x}}=\frac{c \dot{x}}{\eta \sqrt{\dot{x}^{2}-1}} \equiv p_{x}
$$

Using this, we obtain a first-order ordinary differential equation

$$
\frac{d \eta}{d x}=\sqrt{1-\frac{c^{2}}{p_{x}^{2} \eta^{2}}}
$$


which can be easily solved to give the analytic expression for the geodesic

$$
\left\{\begin{array}{l}
\eta(x)=-\sqrt{x^{2}+\frac{c^{2}}{p_{x}^{2}}} \\
y(x)=y_{0}
\end{array}\right.
$$

The holographic screen of FRW universes in the de Sitter limit lies on

$$
\eta=-\sqrt{x^{2}+y^{2}} \equiv-r
$$

Consider a leaf at $\eta=\eta_{*}=-r_{*}$ and a subregion on it specified by a half opening angle $\psi(0 \leq \psi \leq \pi)$. The end points of the HRRT surface are then at

$$
(x, y)=\left(\mp \eta_{*} \sin \psi,-\eta_{*} \cos \psi\right) .
$$

This can be used to determine $p_{x}$ and $y_{0}$ in Eq. (C23), giving the final expression for the geodesic

$$
\left\{\begin{array}{l}
\eta(x)=-\sqrt{x^{2}+y_{0}^{2}} \\
y(x)=y_{0}
\end{array}\right.
$$

where $y_{0}=-\eta_{*} \cos \psi$. By varying the angle $\psi$, the HRRT surfaces sweep a codimension-1 surface in the bulk, which is indeed the future boundary of the causal region of the leaf,

$$
\eta=-r, \quad 0 \leq r \leq r_{*}\left(=-\eta_{*}\right) .
$$

These surfaces are depicted in $x-y-\eta$ space in Fig. 13 . We can clearly see that all the HRRT surfaces are spacelike, except for that corresponding to $\psi=\pi / 2$ which is null.

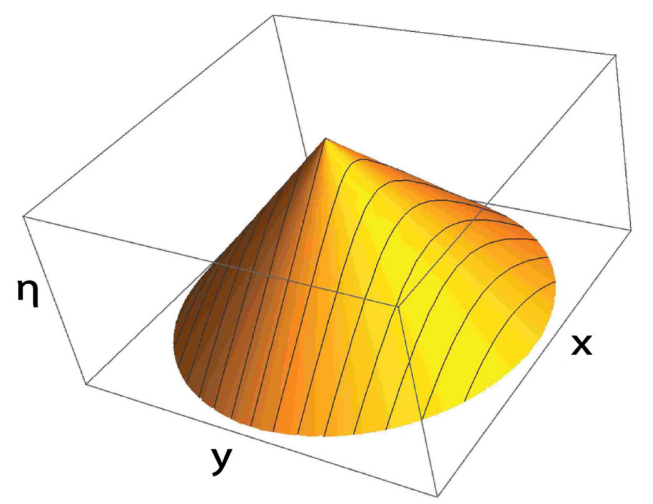

FIG. 13. HRRT surfaces anchored to subregions on a leaf in $(2+1)$-dimensional de Sitter space. They all lie on the future boundary of the causal region associated with the leaf.

\section{b. Small spherical caps in FRW universes in $d+1$ dimensions}

Another example in which simple analytic expressions are obtained is the limit of small spherical cap regions, $\psi \ll 1$, on a leaf. Consider a flat FRW universe in $d+1$ dimensions,

$$
d s^{2}=a(\eta)^{2}\left(-d \eta^{2}+d r^{2}+r^{2} d \Omega_{d-1}^{2}\right),
$$

filled with a single fluid component with the equation of state $w$. We consider the leaf $\sigma_{*}$ at $\eta=\eta_{*}$, which is located at

$$
r=\frac{a}{\dot{a}} .
$$

The future boundary $F_{*}$ of the causal region $D_{\sigma_{*}}$ is then given by

$$
F_{*}: \eta(r)=\eta_{*}+\frac{a}{\dot{a}}-r .
$$

Here and below, the scale factor and its derivatives without an argument represent those at $\eta=\eta_{*}$,

$$
a \equiv a\left(\eta_{*}\right),\left.\quad \dot{a} \equiv \frac{d a(\eta)}{d \eta}\right|_{\eta=\eta_{*}},\left.\quad \ddot{a} \equiv \frac{d^{2} a(\eta)}{d \eta^{2}}\right|_{\eta=\eta_{*}} .
$$

We consider a spherical cap region $A$ on the leaf $\sigma_{*}$, specified by a half opening angle $\psi$,

$$
0 \leq \theta \leq \psi,
$$

where $\theta$ is a polar angle parametrizing $S^{d-1}$ with constant $\eta$ and $r$. Following Ref. [5], we go to cylindrical coordinates,

$$
\xi=r \sin \theta, \quad z=r \cos \theta-\frac{a}{\dot{a}} \cos \psi .
$$

In these coordinates, the null cone $F_{*}$ in Eq. (C30) is given by

$$
F_{*}: \eta(\xi)=\eta_{*}+\frac{a}{\dot{a}}-\sqrt{\xi^{2}+\left(z+\frac{a}{\dot{a}} \cos \psi\right)^{2}},
$$

and the boundary of $A, \partial A$, is located at

$$
\eta=\eta_{*}, \quad \xi=\frac{a}{\dot{a}} \sin \psi \equiv \xi_{*}, \quad z=0 .
$$

The HRRT surface $\gamma_{A}$ anchored to $\partial A$ is on the $z=0$ hypersurface [5]. We compare this HRRT surface with the intersection of $F_{*}$ and $z=0$,

$$
l_{A}: \eta(\xi)=\eta_{*}+\frac{a}{\dot{a}}-\sqrt{\xi^{2}+\frac{a}{\dot{a}} \cos \psi} ;
$$




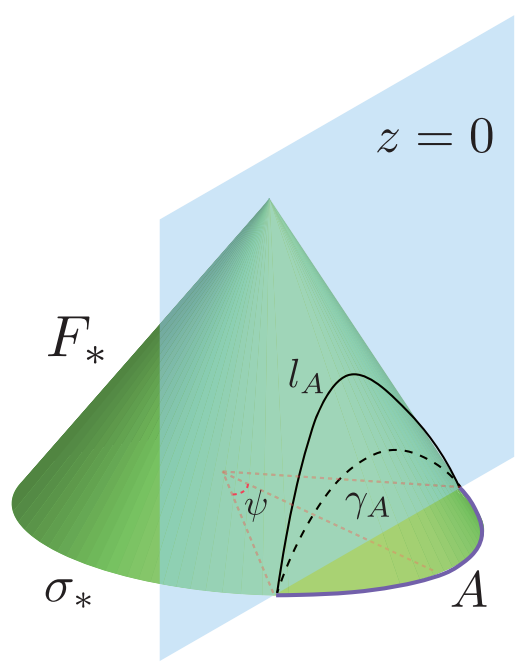

FIG. 14. The HRRT surface $\gamma_{A}$ for subregion $A$ of a leaf $\sigma_{*}$ specified by a half opening angle $\psi$ is on the $z=0$ hypersurface. It approaches the surface $l_{A}$, the intersection of the null cone $F_{*}$ and the $z=0$ hypersurface, in the de Sitter limit.

see Fig. 14. Using Eq. (C35) and expanding in powers of $\psi \sim \xi /(a / \dot{a})$, this can be written as

$l_{A}: \eta(\xi)=\eta_{*}+\frac{\dot{a}}{2 a}\left(\xi_{*}^{2}-\xi^{2}\right)+\frac{\dot{a}^{3}}{8 a^{3}}\left(\xi_{*}^{2}-\xi^{2}\right)^{2}+\cdots$.

For $\psi \ll 1$, the HRRT surface can be expressed in a power series form

$$
\gamma_{A}: \eta(\xi)=\eta_{*}+\eta^{(2)}(\xi)+\eta^{(4)}(\xi)+\cdots
$$

where

$$
\begin{aligned}
& \eta^{(2)}(\xi)=\frac{\dot{a}}{2 a}\left(\xi_{*}^{2}-\xi^{2}\right), \\
& \eta^{(4)}(\xi)=- \frac{\dot{a}}{8 a^{3}(d+1)}\left(\xi_{*}^{2}-\xi^{2}\right) \\
& \times\left[\dot{a}^{2}\left\{(d+5) \xi_{*}^{2}-(d-3) \xi^{2}\right\}\right. \\
&\left.-a \ddot{a}\left\{(d+3) \xi_{*}^{2}-(d-1) \xi^{2}\right\}\right] .
\end{aligned}
$$

In the universe dominated by a single fluid component, the scale factor behaves as

$$
a(\eta) \propto \eta^{\frac{2}{d-2+d w}} .
$$

Plugging this into Eq. (C40), we obtain

$$
\begin{aligned}
\eta^{(4)}(\xi)= & \frac{\dot{a}^{3}}{16(d+1) a^{3}}\left(\xi_{*}^{2}-\xi^{2}\right)[\{2-(1+3 w) d \\
& \left.-(1+w) d^{2}\right\} \xi_{*}^{2}-\{2+(3+w) d \\
& \left.\left.-(1+w) d^{2}\right\} \xi^{2}\right] .
\end{aligned}
$$

We find that for $w=-1$, the surface given by Eqs. (C38), (C39), and (C42) agrees with $l_{A}$ in Eq. (C37). Namely, the HRRT surface $\gamma_{A}$ is on the null cone $F_{*}$.

One can see how $\gamma_{A}$ approaches $F_{*}$ as $w \rightarrow-1$ by subtracting Eq. (C38) from Eq. (C37),

$$
\begin{aligned}
\eta^{l_{A}}(\xi)-\eta^{\gamma_{A}}(\xi)= & \frac{\dot{a}^{3}}{16 a^{3}} \frac{d}{d+1}(1+w)\left(\xi_{*}^{2}-\xi^{2}\right)\left\{(d+3) \xi_{*}^{2}\right. \\
& \left.-(d-1) \xi^{2}\right\} \\
\geq & 0 .
\end{aligned}
$$

The inequality is saturated only for $w=-1$ (except at the end points at $\xi=\xi_{*}$ ).
[1] G. 't Hooft, Dimensional reduction in quantum gravity, in Salamfestschrift, edited by A. Ali, J. Ellis, and S. Randjbar-Daemi (World Scientific, Singapore, 1994), p. 284.

[2] L. Susskind, The world as a hologram, J. Math. Phys. (N.Y.) 36, 6377 (1995).

[3] R. Bousso, The holographic principle, Rev. Mod. Phys. 74, 825 (2002).

[4] J. Maldacena, The large N limit of superconformal field theories and supergravity, Int. J. Theor. Phys. 38, 1113 (1999); Adv. Theor. Math. Phys. 2, 231 (1998).

[5] Y. Nomura, N. Salzetta, F. Sanches, and S. J. Weinberg, Toward a holographic theory for general spacetimes, Phys. Rev. D 95, 086002 (2017).
[6] Y. Nomura, P. Rath, and N. Salzetta, Classical spacetimes as amplified information in holographic quantum theories, arXiv: 1705.06283.

[7] N. Bao and A. Chatwin-Davies, Puzzles and pitfalls involving Haar typicality in holography, arXiv: 1708.08561.

[8] F. Sanches and S. J. Weinberg, Boundary dual of bulk local operators, Phys. Rev. D 96, 026004 (2017).

[9] S. Ryu and T. Takayanagi, Holographic Derivation of Entanglement Entropy from AdS/CFT, Phys. Rev. Lett. 96, 181602 (2006).

[10] V.E. Hubeny, M. Rangamani, and T. Takayanagi, A covariant holographic entanglement entropy proposal, J. High Energy Phys. 07 (2007) 062. 
[11] B. Swingle, Entanglement renormalization and holography, Phys. Rev. D 86, 065007 (2012).

[12] M. Van Raamsdonk, Comments on quantum gravity and entanglement, arXiv:0907.2939.

[13] T. Faulkner, A. Lewkowycz, and J. Maldacena, Quantum corrections to holographic entanglement entropy, J. High Energy Phys. 11 (2013) 074.

[14] N. Engelhardt and A. C. Wall, Quantum extremal surfaces: holographic entanglement entropy beyond the classical regime, J. High Energy Phys. 01 (2015) 073.

[15] F. Sanches and S. J. Weinberg, A holographic entanglement entropy conjecture for general spacetimes, Phys. Rev. D 94, 084034 (2016).

[16] D. N. Page, Average Entropy of a Subsystem, Phys. Rev. Lett. 71, 1291 (1993).

[17] A. Almheiri, X. Dong, and D. Harlow, Bulk locality and quantum error correction in AdS/CFT, J. High Energy Phys. 04 (2015) 163.

[18] R. Bousso, Holography in general spacetimes, J. High Energy Phys. 06 (1999) 028.

[19] D. L. Jafferis, A. Lewkowycz, J. Maldacena, and S. J. Suh, Relative entropy equals bulk relative entropy, J. High Energy Phys. 06 (2016) 004.

[20] X. Dong, D. Harlow, and A. C. Wall, Reconstruction of bulk operators within the entanglement wedge in gauge-gravity duality, Phys. Rev. Lett. 117, 021601 (2016).

[21] D. Kabat and G. Lifschytz, Local bulk physics from intersecting modular Hamiltonians, J. High Energy Phys. 06 (2017) 120.

[22] Y. Kusuki, T. Takayanagi, and K. Umemoto, Holographic entanglement entropy on generic time slices, J. High Energy Phys. 06 (2017) 021.

[23] N. Engelhardt and A. C. Wall, Extremal surface barriers, J. High Energy Phys. 03 (2014) 068.

[24] K. Papadodimas and S. Raju, Remarks on the necessity and implications of state-dependence in the black hole interior, Phys. Rev. D 93, 084049 (2016).

[25] F. Pastawski, B. Yoshida, D. Harlow, and J. Preskill, Holographic quantum error-correcting codes: toy models for the bulk/boundary correspondence, J. High Energy Phys. 06 (2015) 149.

[26] P. Hayden, S. Nezami, X.-L. Qi, N. Thomas, M. Walter, and Z. Yang, Holographic duality from random tensor networks, J. High Energy Phys. 11 (2016) 009.

[27] L. Susskind and E. Witten, The holographic bound in antide Sitter space, arXiv:9805114.

[28] L. Randall and R. Sundrum, An Alternative to Compactification, Phys. Rev. Lett. 83, 4690 (1999).

[29] A. C. Wall, Maximin surfaces, and the strong subadditivity of the covariant holographic entanglement entropy, Classical Quantum Gravity 31, 225007 (2014).

[30] M. Miyaji and T. Takayanagi, Surface/state correspondence as a generalized holography, Prog. Theor. Exp. Phys. 2015, 073 B03 (2015).

[31] J. Maldacena, S. H. Shenker, and D. Stanford, A bound on chaos, J. High Energy Phys. 08 (2016) 106.

[32] M. Srednicki, Chaos and quantum thermalization, Phys. Rev. E 50, 888 (1994).

[33] R. Nandkishore and D. A. Huse, Many body localization and thermalization in quantum statistical mechanics, Annu. Rev. Condens. Matter Phys. 6, 15 (2015).
[34] M. R. Mohammadi Mozaffar and A. Mollabashi, Entanglement in Lifshitz-type quantum field theories, J. High Energy Phys. 07 (2017) 120.

[35] J. L. F. Barbón and C. A. Fuertes, Holographic entanglement entropy probes (non)locality, J. High Energy Phys. 04 (2008) 096.

[36] W. Fischler, A. Kundu, and S. Kundu, Holographic entanglement in a noncommutative gauge theory, J. High Energy Phys. 01 (2014) 137.

[37] J. L. Karczmarek and C. Rabideau, Holographic entanglement entropy in nonlocal theories, J. High Energy Phys. 10 (2013) 078.

[38] N. Shiba and T. Takayanagi, Volume law for the entanglement entropy in nonlocal QFTs, J. High Energy Phys. 02 (2014) 033.

[39] W. Fu and S. Sachdev, Numerical study of fermion and boson models with infinite-range random interactions, Phys. Rev. B 94, 035135 (2016).

[40] C. Liu, X. Chen, and L. Balents, Quantum entanglement of the Sachdev-Ye-Kitaev models, arXiv:1709.06259.

[41] S. Sachdev and J. Ye, Gapless Spin Fluid Ground State in a Random, Quantum Heisenberg Magnet, Phys. Rev. Lett. 70, 3339 (1993).

[42] A. Kitaev, A simple model of quantum holography, KITP Program: Entanglement in Strongly Correlated Quantum Matter, http://online.kitp.ucsb.edu/online/entangled15/kitaev/, 2015; http://online.kitp.ucsb.edu/online/entangled15/kitaev2/.

[43] J. Maldacena and D. Stanford, Remarks on the Sachdev-YeKitaev model, Phys. Rev. D 94, 106002 (2016).

[44] Y. Huang and Y. Gu, Eigenstate entanglement in the Sachdev-Ye-Kitaev model, arXiv:1709.09160.

[45] A. Almheiri, X. Dong, and B. Swingle, Linearity of holographic entanglement entropy, J. High Energy Phys. 02 (2017) 074.

[46] S. Goldstein, J. L. Lebowitz, R. Tumulka, and N. Zanghi, Canonical Typicality, Phys. Rev. Lett. 96, 050403 (2006).

[47] S. Popescu, A. J. Short, and A. Winter, The foundations of statistical mechanics from entanglement: individual states vs averages, Nat. Phys. 2, 754 (2006).

[48] A. C. Wall, A proof of the generalized second law for rapidly changing fields and arbitrary horizon slices, Phys. Rev. D 85, 104049 (2012); Erratum 87, 069904(E) (2013).

[49] N. Bao, S. Nezami, H. Ooguri, B. Stoica, J. Sully, and M. Walter, The holographic entropy cone, J. High Energy Phys. 09 (2015) 130.

[50] T. Faulkner and A. Lewkowycz, Bulk locality from modular flow, J. High Energy Phys. 07 (2017) 151.

[51] J. Cotler, P. Hayden, G. Salton, B. Swingle, and M. Walter, Entanglement wedge reconstruction via universal recovery channels, arXiv:1704.05839.

[52] S. M. Carroll and A. Chatwin-Davies, Cosmic equilibration: a holographic no-hair theorem from the generalized second law, Phys. Rev. D 97, 046012 (2018).

[53] V. Balasubramanian and P. Kraus, Spacetime and the Holographic Renormalization Group, Phys. Rev. Lett. 83, 3605 (1999).

[54] Y. Nomura and N. Salzetta, Butterfly velocities for holographic theories of general spacetimes, J. High Energy Phys. 10 (2017) 187. 
[55] L. Susskind, Entanglement is not enough, Fortschr. Phys. 64, 49 (2016).

[56] Y. Nomura, Quantum mechanics, spacetime locality, and gravity, Found. Phys. 43, 978 (2013).
[57] J. M. Maldacena, Eternal black holes in anti-de Sitter, J. High Energy Phys. 04 (2003) 021.

[58] L. Susskind, L. Thorlacius, and J. Uglum, The stretched horizon and black hole complementarity, Phys. Rev. D 48 , 3743 (1993). 